\title{
CODIMENSION 4 SINGULARITIES OF REFLECTIONALLY SYMMETRYC PLANAR VECTOR FIELDS
}

\author{
F. Dumortier And S. IbÁÑ̃z*
}

\begin{abstract}
The paper deals with the topological classification of singularities of vector fields on the plane which are invariant under reflection with respect to a line. As it has been proved in previous papers, such a classification is necessary to determine the different topological types of singularities of vector fields on $\mathbb{R}^{3}$ whose linear part is invariant under rotations. To get the classification we use normal form theory and the blowing-up method.
\end{abstract}

\section{Introduction}

Consider a $C^{\infty}$ vector field $X$ on $\mathbb{R}^{2}$ with $X(0)=0$. The set of all $C^{\infty}$ vector fields which coincide with $X$ in some neighbourhood of 0 is called the germ of $X$ in 0 and denoted by any of its elements. Let $\bar{G}^{2}$ be the set of all germs in 0 having a representative $X$ which is invariant under the symmetry $R(x, y)=(-x, y)$. We say that $X$ is reflectionally symmetric. In the sequel we assume that each representative which is given for a germ in $\bar{G}^{2}$ is reflectionally symmetric.

Given $X, Y \in \bar{G}^{2}$, they are said to be $k$-jet equivalent, with $k \in \mathbb{N} \cup\{\infty\}$, if their Taylor approximations up to order $k$ are equal. The set of all germs which are $k$-jet equivalent to $X \in \bar{G}^{2}$ is called the $k$ jet of $X$ at 0 and denoted by $j_{k} X(0) . \bar{J}_{k}^{2}$ denotes the set of $k$-jets in $\bar{G}^{2}$.

*Partially supported by the D.G.E.S. Project PB95-1054. 
Let us notice that there exists a one to one correspondence between $\bar{J}_{k}^{2}$ and the space of reflectionally symmetric vector fields $Y$ on $\mathbb{R}^{2}$ with $Y(0)=0$ and whose components are polynomials of degree less or equal than $k$. Therefore we will consider $\bar{J}_{k}^{2}$ as a vector space of finite dimension with the usual topology.

Take the projections $j_{k}: \bar{G}^{2} \rightarrow \bar{J}_{k}^{2}$ induced by the $k$-jet equivalence. $\bar{G}^{2}$ is considered to be endowed with the coarsest topology which makes $j_{k}$ continuous for all $k \in \mathbb{N}$.

A set $A \subset \bar{G}^{2}$ is said to be algebraic if $A=j_{k}^{-1}(\widetilde{A})$ with $\widetilde{A} \subset \bar{J}_{k}^{2}$ an algebraic set of $\bar{J}_{k}^{2}$. The codimension of $A$ is the codimension of $\widetilde{A}$ in $\bar{J}_{k}^{2}$.

On the other hand $X, Y \in \bar{G}^{2}$ are said to be $C^{0}$ equivalent if for some (and hence for all) representatives $\widetilde{X}$ and $\widetilde{Y}$ of $X$ and $Y$, respectively, there are open neighbourhoods $U$ and $V$ of $0 \in \mathbb{R}^{2}$ and a homeomorphism $h: U \rightarrow V$ which sends orbits of $\widetilde{X}$ to orbits of $\widetilde{Y}$ preserving the sense but not necessarily the parametrization.

To give the topological classification of the singularities having a codimension at most four in $\bar{G}^{2}$, we will define a stratification:

$$
\bar{G}^{2}=V_{0} \supset V_{1} \supset V_{2} \supset V_{3} \supset V_{4} \supset V_{5},
$$

where for each $i=1,2,3,4,5, V_{i}$ is a closed algebraic set of codimension $i$ and $V_{i-1} \backslash V_{i}$ is a regular manifold of codimension $i-1$ such that for all $X \in V_{i-1} \backslash V_{i}$ there is a neighbourhood $U$ of $X$ in $\bar{G}^{2}$ for which $Y \in\left(V_{i-1} \backslash V_{i}\right) \cap U$ implies that $X$ and $Y$ are $C^{0}$ equivalent.

Consider $X_{R} \in \bar{G}^{2}$ given by

$$
X_{R}=f(x, y) \frac{\partial}{\partial x}+g(x, y) \frac{\partial}{\partial y}
$$

where $f$ and $g$ are $C^{\infty}$ functions. Since $X_{R}$ is reflectionally symmetric, it follows that $f(-x, y)=-f(x, y)$ and $g(-x, y)=g(x, y)$. As a consequence of the Malgrange Division Theorem $[\mathbf{C H}]$ we know that there exist $C^{\infty}$ functions $\tilde{f}, \tilde{g}: \mathbb{R}^{2} \rightarrow \mathbb{R}$ such that

$$
f(x, y)=x \tilde{f}\left(x^{2}, y\right), \quad g(x, y)=\tilde{g}\left(x^{2}, y\right) .
$$


Therefore, we can use the change of coordinates $w=x^{2}$ and $z=y$ to write

$$
X_{R}=w \tilde{f}(w, z) \frac{\partial}{\partial w}+\tilde{g}(w, z) \frac{\partial}{\partial z}
$$

Let us now study expression (2) using again $(x, y)$ instead of $(w, z)$ and $f, g$ instead of $\tilde{f}, \tilde{g}$ :

$$
X_{R}=x f(x, y) \frac{\partial}{\partial x}+g(x, y) \frac{\partial}{\partial y} .
$$

We only need to study the restriction of $X_{R}$ to the halfplane $\{(x, y) \in$ $\left.\mathbb{R}^{2}: x \geq 0\right\}$.

Consider the following developments of $f$ and $g$ :

$$
\begin{aligned}
f(x, y)= & a_{00}+a_{10} y+a_{01} x+a_{20} y^{2}+a_{11} y x+a_{02} x^{2}+\circ\left(\|(x, y)\|^{2}\right) \\
g(x, y)= & b_{10} y+b_{01} x+b_{20} y^{2}+b_{11} y x+b_{02} x^{2} \\
& +b_{30} y^{3}+b_{21} y^{2} x+b_{12} y x^{2}+b_{03} x^{3}+\circ\left(\|(x, y)\|^{3}\right) .
\end{aligned}
$$

If $a_{00} \neq 0$ and $b_{10} \neq 0$ the singularity is hyperbolic. Therefore, such conditions define the codimension 0 singularities and their corresponding phase-portraits are well known. The same happens with the semihyperbolic cases. They correspond to one of the following conditions

$$
a_{00} \neq 0, \quad b_{10}=0
$$

or

$$
a_{00}=0, \quad b_{10} \neq 0 .
$$

To determine the subsequent stratification we use the reduction to a 1-dimensional center manifold. To draw the different topological types one only needs to consider the classification of the semihyperbolic singularities on the plane up to codimension 5 (see $[\mathbf{D}]$ ), bearing in mind that the $y$-axis is always invariant. Therefore we will find singularities which are topologically equivalent to either saddles or nodes or saddle-nodes.

We will start the classification on a stratum $W_{2} \subset V_{2}$ of codimension 2 given by the conditions

$$
a_{00}=0, \quad b_{10}=0
$$


Hence (3) reduces to

$$
\begin{aligned}
X_{R}= & x\left(a_{10} y+a_{01} x+a_{20} y^{2}+a_{11} y x+a_{02} x^{2}+\circ\left(\|(x, y)\|^{2}\right)\right) \frac{\partial}{\partial x} \\
& +\left(b_{01} x+b_{20} y^{2}+b_{11} y x+b_{02} x^{2}\right. \\
& \left.+b_{30} y^{3}+b_{21} y^{2} x+b_{12} y x^{2}+b_{03} x^{3}+\circ\left(\|(x, y)\|^{3}\right)\right) \frac{\partial}{\partial y} .
\end{aligned}
$$

Moreover we will distinguish two cases:

$$
\begin{array}{ll}
b_{01} \neq 0 & \text { (nilpotent case) } \\
b_{01}=0 & \text { (quadratic case) } .
\end{array}
$$

They will be studied in sections 2 and 3, respectively. For each of them we will consider aditional restrictions. Although the study of the nilpotent case for codimension 2 is already present in the literature (see $[\mathbf{T}]$ ) and also some singularities of codimension 3 in the quadratic case are considered in $[\mathbf{K R}]$, we include them here for the sake of completeness.

Our main motivation for the study of the topological classification of reflectionally symmetric planar vector fields is that they play an essential role in the classification of singularities in $\mathbb{R}^{3}$. As it is shown in $[\mathbf{T}]$, any $C^{\infty}$ vector field $X$ on $\mathbb{R}^{3}$, with $X(0)=0$ and 1-jet linearly conjugate to the infinitesimal rotation $\lambda\left(y \frac{\partial}{\partial x}-x \frac{\partial}{\partial y}\right)$, can be given in a normal form which is, up to flat terms, invariant under rotations. After normalizing the rotational component and introducing cylindrical coordinates $(\theta, r, z), X$ can be written as

$$
2 \pi \frac{\partial}{\partial \theta}+X_{R}+Y
$$

where $j_{\infty} Y(\theta, 0,0)=0$ for all $\theta \in S^{1}$ and $X_{R}$ is a reflectionally symmetric planar vector field, depending on the coordinates $(r, z)$, such that $X_{R} \in W_{2}$. In $[\mathbf{D I}]$ it is shown that, at least up to codimension four, flat terms have no influence on the topological type of the singularity which, in fact, is given by the topological type of $X_{R}$. Nevertheless, neither the classification of the reflectionally symmetric vector fields $X_{R}$ nor the topological types were described in $[\mathbf{D I}]$. In this paper we provide such a complete classification. The pictures that we give for the phase portraits have been made with the computer package $\mathrm{P} 4$ (see $[\mathbf{D H}]$ and $[\mathbf{H}]$ ). The models which are used will be indicated in each case. We would like to thank Chris Herssens and Peter de Maesschalck for having helped us in making these pictures. 


\section{Nilpotent case $\left(b_{01} \neq 0\right)$}

A linear coordinate change permits to take $b_{01}=1$. Hence we can write the $\infty$-jet of $X_{R}$ as

$$
\left(\sum_{i+j \geq 1} a_{i j} y^{i} x^{j}\right) x \frac{\partial}{\partial x}+\left(x+\sum_{i+j \geq 2} b_{i j} y^{i} x^{j}\right) \frac{\partial}{\partial y}
$$

One can prove that, up to a $C^{\infty}$ coordinate change which leaves $\{x=0\}$ invariant, the $\infty$-jet of $X_{R}$ can be written as:

$$
x\left(\sum_{i \geq 1} a_{i} y^{i}\right) \frac{\partial}{\partial x}+\left(x+\sum_{i \geq 2} b_{i} y^{i}\right) \frac{\partial}{\partial y} .
$$

Indeed, let $m \geq 2$ be an integer. Since

$$
\left[x \frac{\partial}{\partial y}, x^{j-1} y^{m-j+1} \frac{\partial}{\partial x}\right]=(m-j+1) x^{j} y^{m-j} \frac{\partial}{\partial x}-x^{j-1} y^{m-j+1} \frac{\partial}{\partial y},
$$

all the terms $x^{j} y^{m-j} \frac{\partial}{\partial x}$ with $j \geq 2$ can be removed after a change of coordinates which does not affect neither the remaining terms of the $m$-jet of the $\frac{\partial}{\partial x}$-component nor the terms of the $(m-1)$-jet of the $\frac{\partial}{\partial y}$-component. Moreover, since

$$
\left[x \frac{\partial}{\partial y}, x^{j-1} y^{m-j+1} \frac{\partial}{\partial y}\right]=(m-j+1) x^{j} y^{m-j} \frac{\partial}{\partial y},
$$

all the terms $x^{j} y^{m-j} \frac{\partial}{\partial y}$ with $j \geq 1$ can be removed after a change of coordinates which does not affect the remaining terms of the $m$-jet of the vector field. All the changes of coordinates which are needed leave $\{x=0\}$ invariant and it is important to observe that (5) is the simplest normal form which preserves such an invariance. 
We define the following stratification

$$
\begin{aligned}
& N_{2,1}=\left\{X \in W_{2} \mid b_{01} \neq 0\right\}, \\
& N_{3,1}=\left\{X \in N_{2,1} \mid b_{2}=0\right\}, \\
& N_{3,2}=\left\{X \in N_{2,1} \mid b_{2} \neq 0, a_{1}=0\right\}, \\
& N_{3,3}=\left\{X \in N_{2,1} \mid b_{2} \neq 0, a_{1} \neq 0, a_{1}=2 b_{2}\right\}, \\
& N_{4,1}=\left\{X \in N_{2,1} \mid b_{2}=0, a_{1}=0\right\}, \\
& N_{4,2}=\left\{X \in N_{2,1} \mid b_{2}=0, a_{1} \neq 0, b_{3}=0\right\}, \\
& N_{4,3}=\left\{X \in N_{2,1} \mid b_{2} \neq 0, a_{1}=0, a_{2}=0\right\}, \\
& N_{5,1}=\left\{X \in N_{2,1} \mid b_{2}=0, a_{1}=0, a_{2}=0\right\}, \\
& N_{5,2}=\left\{X \in N_{2,1} \mid b_{2}=0, a_{1}=0, a_{2} \neq 0, b_{3}=0\right\}, \\
& N_{5,3}=\left\{X \in N_{2,1} \mid b_{2}=0, a_{1}=0, a_{2} \neq 0, b_{3} \neq 0, a_{2}=3 b_{3}\right\}, \\
& N_{5,4}=\left\{X \in N_{2,1} \mid b_{2}=0, a_{1} \neq 0, b_{3}=0, b_{4}=0\right\}, \\
& N_{5,5}=\left\{X \in N_{2,1} \mid b_{2} \neq 0, a_{1}=0, a_{2}=0, a_{3}=0\right\} .
\end{aligned}
$$

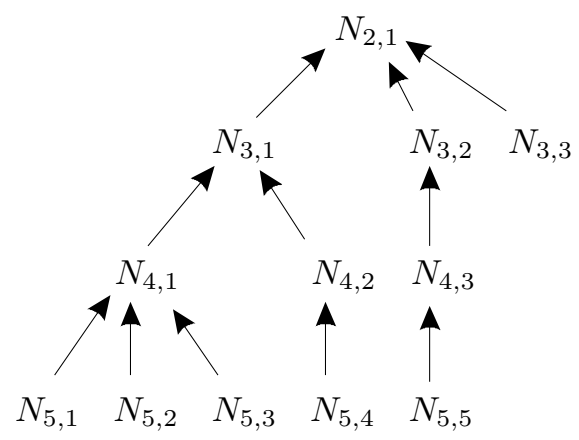

Figure 2.1. Stratification in the nilpotent case.

Note that each $N_{i, j}$ with $i=2,3,4,5$ is a semi-algebraic set of codimension $i$. In Figure 2.1 we show how the previous strata are organized. Let us now consider the different equivalence classes which are found in each stratum. To do this we use the blow-up

$$
x=r^{2} u, \quad y=r v
$$


with $u^{2}+v^{2}=1$ and $r \in \mathbb{R}^{+}$and as usual the calculations are made by means of directional blow-up's. Since the exponent of $r$ in the change $y=r v$ is odd the vector field obtained by blowing-up in the $y$-direction with $v=-1$ is related with the one obtained by taking $v=1$ by means of the change $(r, u) \rightarrow(-r, u)$ and the rescaling $t \rightarrow-t$. We keep this in mind in order to draw the different phase-portraits after blowing up. Further simplifications are possible in each case separately.

Remark 2.1. Let us recall that in the previous observations and calculations made on expression $(3)$ the coordinates $(x, y)$ represent in fact $(w, z)$ in expression (2). Going back to the original coordinates $(x, y)$ in (1) we have to use $w=x^{2}$ and $z=y$. Now by means of blow-up we will, in many cases, prove the existence of invariant curves for vector fields like in (2) of the form $w=\alpha z^{2}+o\left(z^{2}\right)$ with $\alpha$ positive. Hence, with respect to the original $(x, y)$-coordinates, we get invariant curves of the form $x=\sqrt{\alpha} y+o(y)$ for vector fields (1). However, in the cases drawn in Figure 2.9, the invariant curves in $\{x>0\}$ are obtained by a blow-up $\left(w=r^{3} u, z=r v\right)$ and will be of the form $x=\gamma y^{3 / 2}+o\left(y^{3 / 2}\right)$ for some $\gamma>0$.

\subsection{Singularities of codimension 2 .}

Let $X \in N_{2,1} \backslash\left(N_{3,1} \cup N_{3,2} \cup N_{3,3}\right)$. In this case we have $b_{2} \neq 0, a_{1} \neq 0$ and $a_{1} \neq 2 b_{2}$. By means of the change of variables $x=\bar{x} / b_{2}, y=\bar{y} / b_{2}$, we get from (5) the vector field

$$
\left\{\begin{array}{l}
\bar{x}^{\prime}=\frac{a_{1}}{b_{2}} \bar{x} \bar{y}+\bar{x} \sum_{i \geq 2} \frac{a_{i}}{b_{2}^{i}} \bar{y}^{i} \\
\bar{y}^{\prime}=\bar{x}+\bar{y}^{2}+\sum_{j \geq 3} \frac{b_{j}}{b_{2}^{j-1}} \bar{y}^{j} .
\end{array}\right.
$$

Blowing-up in the $\bar{y}$-direction with $v=1$, we get

$$
\left\{\begin{array}{l}
r^{\prime}=r u+r+o(r) \\
u^{\prime}=\left(\frac{a_{1}}{b_{2}}-2\right) u-2 u^{2}+u(O(r)) .
\end{array}\right.
$$

This vector field restricted to $\{r=0\}$ has exactly two hyperbolic singularities at $(r, u)=(0,0)$ and $(r, u)=\left(0,\left(a_{1} / b_{2}-2\right) / 2\right)$. Blowing-up in the $\bar{x}$-direction, no singularity is found at $(r, v)=(0,0)$. The study of the singularities leads to the phase-portraits given in Figure 2.2 (a) for a neighbourhood of $S^{1} \times\{0\}$. In Figure 2.2 (b) we represent the different 
topological types corresponding to $X_{R}$ as in (1). Note that, because of the change of variables used to get (6), we must take into account the sign of $b_{2}$.

(a)
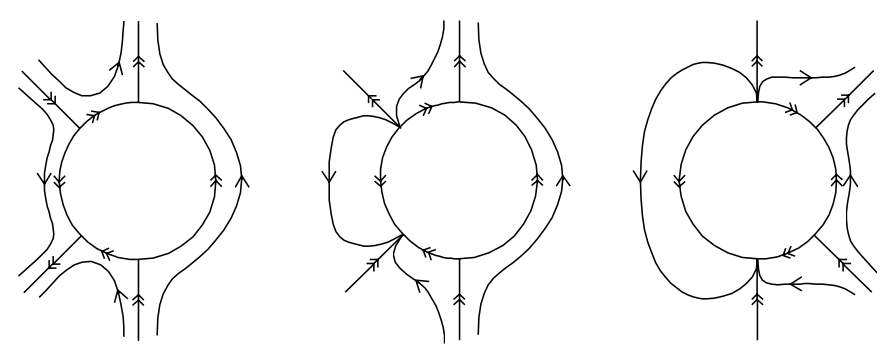

$$
\frac{a_{1}}{b_{2}}<0
$$

$$
0<\frac{a_{1}}{b_{2}}<2
$$

$$
2<\frac{a_{1}}{b_{2}}
$$

(b)
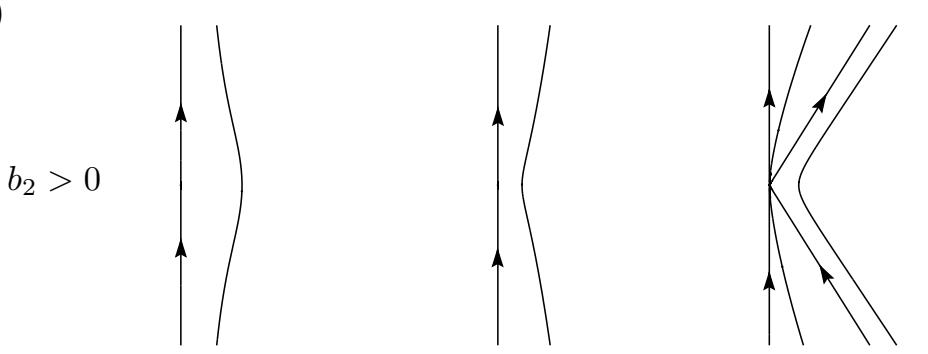

$$
\left(a_{1}, b_{2}\right)=(-1,1)
$$

$\left(a_{1}, b_{2}\right)=(1,1)$
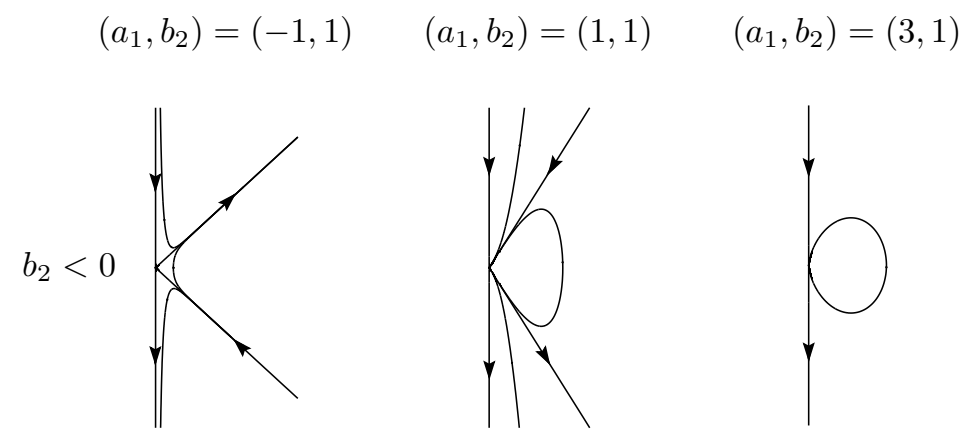

$$
\begin{array}{ccc}
\left(a_{1}, b_{2}\right)=(1,-1) & \left(a_{1}, b_{2}\right)=(-1,-1) & \left(a_{1}, b_{2}\right)=(-3,-1) \\
\frac{a_{1}}{b_{2}}<0 & 0<\frac{a_{1}}{b_{2}}<2 & 2<\frac{a_{1}}{b_{2}}
\end{array}
$$

Figure 2.2. Topological types for vector fields in $N_{2,1} \backslash\left(N_{3,1} \cup N_{3,2} \cup N_{3,3}\right)$. We use the model $\frac{a_{1}}{2} x y \frac{\partial}{\partial x}+\left(x^{2}+b_{2} y^{2}\right) \frac{\partial}{\partial y}$. 
Before continuing with the study of the singularities of higher codimension let us quickly describe the dynamic degeneracies that will occur in each of the next strata. For that we look at the singularities which appear after the previous directional blowing-up. For vector fields in $N_{3,1}$ the singularity at $(0,0)$ has a vanishing radial eigenvalue because of the coalescence with an extra hyperbolic singular point. Along the branches $N_{3,1}, N_{4,2}$ and $N_{5,4}$ new singular points coalesce with $(0,0)$ leading to more degenerate singularities, however staying semi-hyperbolic in all cases. A similar phenomenon happens at $\left(0,\left(a_{1} / b_{2}-2\right) / 2\right)$ for the branches $N_{3,2}, N_{4,3}$ and $N_{5,5}$. For vector fields in $N_{3,3}$ both singularities on the blown-up circle $\{r=0\}$ coalesce at $(0,0)$ which hence becomes a semi-hyperbolic singular point whose center manifold is contained in $\{r=0\}$; further degeneracies are not possible. Something similar happens for $N_{4,1}$ but in this case the singularity at $(0,0)$ has a double zero eigenvalue and an extra blowing-up should be used. We will find again two singularities on the new blown-up circle. Then the strata $N_{5, j}$ with $j=1,2,3$ will have the same dynamical meaning, with respect to these new singularities, as $N_{3, j}$ has with respect to those obtained after the first blowing-up. In any case, as we will see later, it is better to modify the original blowing-up instead of making an extra one. On the other hand, looking at the quadratic case as limit of the nilpotent case, when $b_{01}=0$, and applying the same blowing-up, we would find degenerate singularities on the second blown-up circle. This is the reason why we use, after further simplifications, a different blowing-up. If we take into account the influence of $b_{01}$, the singularity $\left(0,\left(a_{1} / b_{2}-2\right) / 2\right)$ will tend to infinity when $b_{01}$ tends to 0 , leading to the coalescence of two singular points on the blown-up circle. Such degeneracy is considered in the quadratic case.

\subsection{Singularities of codimension 3 .}

We have three different cases:

1. $X \in N_{3,3}$. In this case $b_{2} \neq 0, a_{1} \neq 0$ and $a_{1}=2 b_{2}$,

2. $X \in N_{3,2} \backslash N_{4,3}$. In this case $b_{2} \neq 0, a_{1}=0$ and $a_{2} \neq 0$,

3. $X \in N_{3,1} \backslash\left(N_{4,1} \cup N_{4,2}\right)$. In this case $b_{2}=0, a_{1} \neq 0$ and $b_{3} \neq 0$, which are studied separately.

Case 1: Since $b_{2} \neq 0$ we use (6) which is given, taking into account that $a_{1}=2 b_{2}$, by

$$
\left\{\begin{array}{l}
\bar{x}^{\prime}=2 \bar{x} \bar{y}+\bar{x} \sum_{i \geq 2} \frac{a_{i}}{b_{2}^{i}} \bar{y}^{i} \\
\bar{y}^{\prime}=\bar{x}+\bar{y}^{2}+\sum_{j \geq 3} \frac{b_{j}}{b_{2}^{j-1}} \bar{y}^{j}
\end{array}\right.
$$


Blowing-up in the $\bar{y}$-direction with $v=1$, we get

$$
\left\{\begin{array}{l}
r^{\prime}=r u+r+o(r) \\
u^{\prime}=-2 u^{2}+u(O(r)) .
\end{array}\right.
$$

On the invariant line $\{r=0\}$ we find exactly one singularity at $(r, u)=(0,0)$. The linear part at $(0,0)$ has eigenvalues 1 and 0 . The unstable manifold is contained in $\{u=0\}$ while $\{r=0\}$ contains a center manifold. One can check that blowing-up in the $\bar{x}$-direction no singularity is found at $(r, v)=(0,0)$. Glueing the different charts together we obtain the phase-portrait given in Figure 2.3 (a) for a neighbourhood of $S^{1} \times\{0\}$. In Figure 2.3 (b) we represent the different topological types corresponding to $X_{R}$. Again we must take into account the sign of $b_{2}$.

(a)

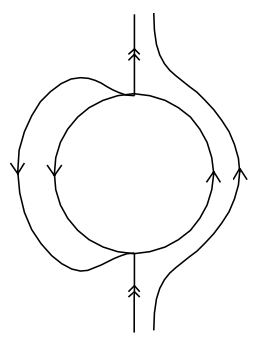

(b)

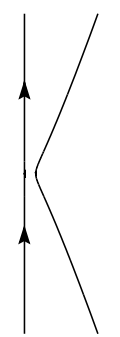

$b_{2}=1$

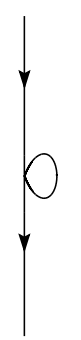

$b_{2}=-1$

$b_{2}>0, a_{1}=2 b_{2} \quad b_{2}<0, a_{1}=2 b_{2}$

Figure 2.3. Topological types for vector fields in $N_{3,3}$. We use the model $b_{2} x y \frac{\partial}{\partial x}+\left(x^{2}+b_{2} y^{2}\right) \frac{\partial}{\partial y}$.

Case 2: We use again (6) which is given, taking into account that $a_{1}=0$, by

$$
\left\{\begin{array}{l}
\bar{x}^{\prime}=\frac{a_{2}}{b_{2}^{2}} \bar{x} \bar{y}^{2}+\bar{x} \sum_{i \geq 3} \frac{a_{i}}{b_{2}^{i}} \bar{y}^{i} \\
\bar{y}^{\prime}=\bar{x}+\bar{y}^{2}+\frac{b_{3}}{b_{2}^{2}} \bar{y}^{3}+\sum_{j \geq 4} \frac{b_{j}}{b_{2}^{j-1}} \bar{y}^{j} .
\end{array}\right.
$$

Blowing-up in the $\bar{y}$-direction with $v=1$, we get

$$
\left\{\begin{array}{l}
r^{\prime}=r u+r+\frac{b_{3}}{b_{2}^{2}} r^{2}+o\left(r^{2}\right) \\
u^{\prime}=-2 u(u+1)+\left(\frac{a_{2}}{b_{2}^{2}}-\frac{2 b_{3}}{b_{2}^{2}}\right) r u+u(o(r)) .
\end{array}\right.
$$


On the invariant line $\{r=0\}$ we find exactly two singularities at $(r, u)=(0,0)$ and $(r, u)=(0,-1)$. The linear part at $(0,0)$ has eigenvalues 1 and -2 and $\{r=0\}$ (resp. $\{u=0\}$ ) contains the stable (resp. unstable) manifold at $(0,0)$. The linear part at $(0,-1)$ has eigenvalues 0 and 2. In this case $\{r=0\}$ contains the unstable manifold. Putting $u+1=z$, we bring the singularity at $(0,-1)$ to $(r, z)=(0,0)$. With respect to the new coordinates a first order approximation of the center manifold is given by $z=\left(a_{2} / 2 b_{2}^{2}-b_{3} / b_{2}^{2}\right) r+o(r)$. Hence, the reduction at order 2 to the center manifold is

$$
r^{\prime}=\frac{a_{2}}{2 b_{2}^{2}} r^{2}+o\left(r^{2}\right) .
$$

Blowing-up in the $\bar{x}$-direction no singularity is found at $(r, v)=(0,0)$. The phase-portraits for the blowing-up are given in Figure 2.4 (a). Taking into account the sign of $b_{2}$ we obtain the topological types depicted in Figure 2.4 (b) for $X_{R}$.

(a)
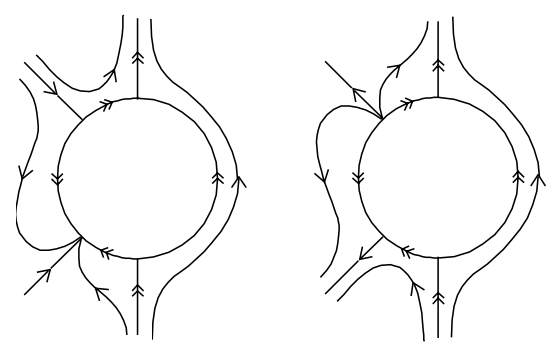

$$
a_{2}<0
$$

$$
a_{2}>0
$$

(b)

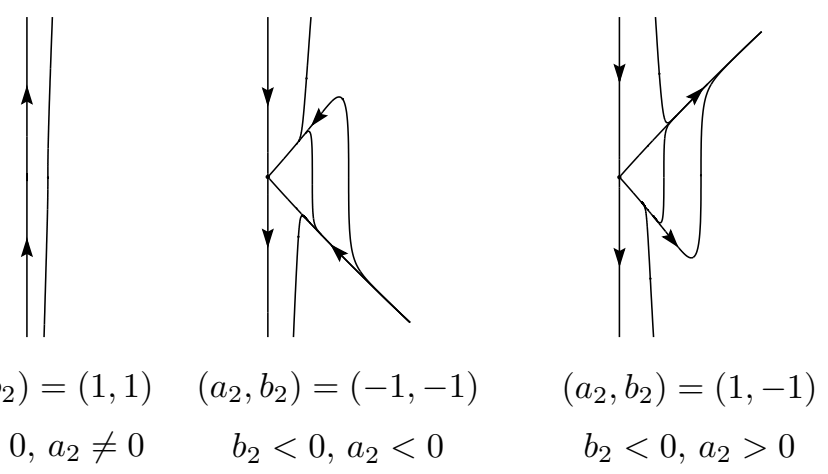

Figure 2.4. Topological types for vector fields in $N_{3,2} \backslash N_{4,3}$. We use the model $\frac{a_{2}}{2} x y^{2} \frac{\partial}{\partial x}+\left(x^{2}+b_{2} y^{2}\right) \frac{\partial}{\partial y}$. 
Case 3: By means of the change of variables and the rescaling given by

$$
\left\{\begin{array}{l}
x=\frac{a_{1}^{3}}{b_{3}^{2}} \bar{x} \\
y=\frac{a_{1}}{b_{3}} \bar{y}
\end{array}\right.
$$

we get from (5) the vector field

$$
\left\{\begin{array}{l}
\bar{x}^{\prime}=\bar{x}(\bar{y}+o(\bar{y})) \\
\bar{y}^{\prime}=\bar{x}+\bar{y}^{3}+o\left(\bar{y}^{3}\right) .
\end{array}\right.
$$

Blowing-up in the $\bar{y}$-direction with $v=1$, we get

$$
\left\{\begin{array}{l}
r^{\prime}=r u+r^{2}+o\left(r^{2}\right) \\
u^{\prime}=u(1-2 u)+u(O(r)) .
\end{array}\right.
$$

(a)

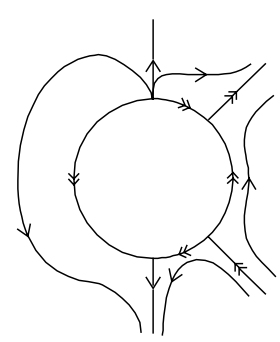

(b)

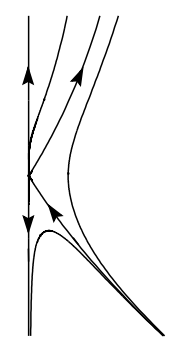

$$
\begin{array}{cc}
\left(a_{1}, b_{3}\right)=(1,1) & \left(a_{1}, b_{3}\right)=(1,-1) \\
a_{1}>0, b_{3}>0 & a_{1}>0, b_{3}<0
\end{array}
$$
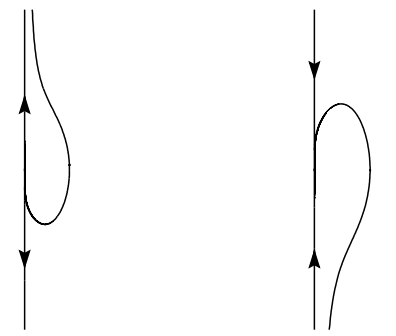

$$
\begin{array}{cc}
\left(a_{1}, b_{3}\right)=(-1,1) & \left(a_{1}, b_{3}\right)=(-1,-1) \\
a_{1}<0, b_{3}>0 & a_{1}<0, b_{3}<0
\end{array}
$$

Figure 2.5. Topological types for vector fields in $N_{3,1} \backslash\left(N_{4,1} \cup N_{4,2}\right)$. We use the model $\frac{a_{1}}{2} x y \frac{\partial}{\partial x}+\left(x^{2}+b_{3} y^{3}\right) \frac{\partial}{\partial y}$. 
On the invariant line $\{r=0\}$ we find exactly two singularities at $(r, u)=(0,0)$ and $(r, u)=(0,1 / 2)$. The linear part at $(0,0)$ has eigenvalues 0 and 1 and $\{r=0\}$ (resp. $\{u=0\}$ ) contains the unstable manifold (resp. a center manifold). The linear part at $(0,1 / 2)$ has eigenvalues $1 / 2$ and -1 . Again all the relevant information is given by the previous blowing-up. Figure 2.5 shows the phase-portrait close to $S^{1} \times\{0\}$ and the corresponding flows for $X_{R}$, which are obtained taking into account the signs of $a_{1}$ and $b_{3}$ in (7).

\subsection{Singularities of codimension 4 .}

We will distinguish four cases:

1. $X \in N_{4,3} \backslash N_{5,5}$. In this case $b_{2} \neq 0, a_{1}=0, a_{2}=0$ and $a_{3} \neq 0$,

2. $X \in N_{4,2} \backslash N_{5,4}$. In this case $b_{2}=0, a_{1} \neq 0, b_{3}=0$ and $b_{4} \neq 0$,

3. $X \in N_{4,1} \backslash\left(N_{5,1} \cup N_{5,2} \cup N_{5,3}\right)$. In this case $b_{2}=0, a_{1}=0, a_{2} \neq 0$, $b_{3} \neq 0$ and $a_{2} \neq 3 b_{3}$,

which are studied separately.

Case 1: Since $b_{2} \neq 0$ we use again (6) which is given, taking into account that $a_{1}=a_{2}=0$, by

$$
\left\{\begin{array}{l}
\bar{x}^{\prime}=\frac{a_{3}}{b_{2}^{3}} \bar{x} \bar{y}^{3}+\bar{x} \sum_{i \geq 4} \frac{a_{i}}{b_{2}^{i}} \bar{y}^{i} \\
\bar{y}^{\prime}=\bar{x}+\bar{y}^{2}+\frac{b_{3}}{b_{2}^{2}} \bar{y}^{3}+\frac{b_{4}}{b_{2}^{3}} \bar{y}^{4}+\sum_{j \geq 5} \frac{b_{j}}{b_{2}^{j-1}} \bar{y}^{j} .
\end{array}\right.
$$

Blowing-up in the $\bar{y}$-direction with $v=1$, we get

$$
\left\{\begin{array}{l}
r^{\prime}=r u+r+\frac{b_{3}}{b_{2}^{2}} r^{2}+\frac{b_{4}}{b_{2}^{3}} r^{3}+o\left(r^{3}\right) \\
u^{\prime}=-2 u(u+1)-\frac{2 b_{3}}{b_{2}^{2}} r u+\left(\frac{a_{3}}{b_{2}^{3}}-\frac{2 b_{4}}{b_{2}^{3}}\right) r^{2} u+u\left(o\left(r^{2}\right)\right) .
\end{array}\right.
$$

On the invariant line $\{r=0\}$ we find exactly two singularities at $(r, u)=(0,0)$ and $(r, u)=(0,-1)$. The linear part at $(0,0)$ has eigenvalues 1 and -2 and $\{r=0\}$ (resp. $\{u=0\}$ ) contains the stable (resp. unstable) manifold at $(0,0)$. The linear part at $(0,-1)$ has eigenvalues 0 and 2 . In this case $\{r=0\}$ contains the unstable manifold. 
Putting $u+1=z$, we bring the singularity at $(0,-1)$ to $(r, z)=(0,0)$. With respect to the new coordinates a second order approximation of the center manifold is given by $z=\left(-b_{3} / b_{2}^{2}\right) r+\left(a_{3} / 2 b_{2}^{3}-b_{4} / b_{2}^{3}\right) r^{2}+o\left(r^{2}\right)$. Hence, the reduction at order 3 to the center manifold is

$$
r^{\prime}=\frac{a_{3}}{2 b_{2}^{3}} r^{3}+o\left(r^{3}\right)
$$

Blowing-up in the $\bar{x}$-direction no singularity is found at $(r, v)=(0,0)$. The phase-portraits for the blowing-up are given in Figure 2.6 (a). Taking into account the sign of $b_{2}$ we obtain the topological types depicted in Figure 2.6 (b) for $X_{R}$.

(a)
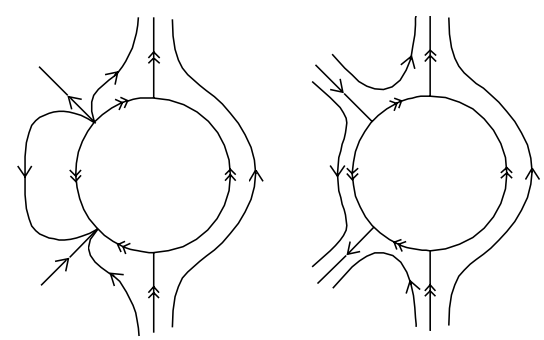

$$
\frac{a_{3}}{2 b_{2}^{3}}>0
$$

$$
\frac{a_{3}}{2 b_{2}^{3}}<0
$$

(b)
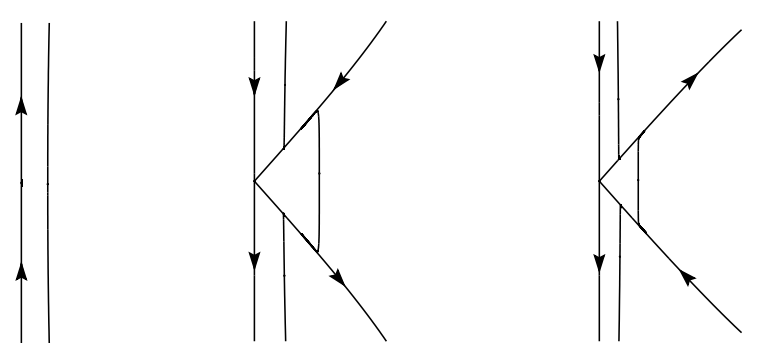

$$
\begin{array}{ccc}
\left(a_{3}, b_{2}\right)=(1,1) & \left(a_{3}, b_{2}\right)=(-1,-1) & \left(a_{3}, b_{2}\right)=(1,-1) \\
b_{2}>0, a_{3} \neq 0 & b_{2}<0, a_{3}<0 & b_{2}<0, a_{3}>0
\end{array}
$$

Figure 2.6. Topological types for vector fields in $N_{4,3} \backslash N_{5,5}$. We use the model $\frac{a_{3}}{2} x y^{3} \frac{\partial}{\partial x}+\left(x^{2}+b_{2} y^{2}\right) \frac{\partial}{\partial y}$. 
Case 2: Suppose that $a_{1} b_{4}>0$. In such a case we use the change of variables and the rescaling given by

$$
\left\{\begin{array}{l}
x=\frac{a_{1}^{2}}{b_{4}} \bar{x} \\
y=\left(\frac{a_{1}}{b_{4}}\right)^{\frac{1}{2}} \bar{y}
\end{array}\right.
$$

to obtain from (5) the vector field

$$
\left\{\begin{array}{l}
\bar{x}^{\prime}=\bar{x} \bar{y}+\bar{x}(o(\bar{y})) \\
\bar{y}^{\prime}=\bar{x}+\bar{y}^{4}+o\left(\bar{y}^{4}\right) .
\end{array}\right.
$$

Blowing-up in the $\bar{y}$-direction with $v=1$, we get

$$
\left\{\begin{array}{l}
r^{\prime}=r u+r^{3}+o\left(r^{3}\right) \\
u^{\prime}=u-2 u^{2}+u(O(r)) .
\end{array}\right.
$$

On the invariant line $\{r=0\}$ we find exactly two singularities at $(r, u)=(0,0)$ and $(r, u)=(0,1 / 2)$. The singularity $(0,1 / 2)$ is hyperbolic and the eigenvalues of the linear part are $1 / 2$ and -1 . The eigenvalues of the linear part at $(0,0)$ are 0 and 1 . The unstable manifold is contained in $\{r=0\}$ and since $\{u=0\}$ is invariant, it contains a center manifold. All the relevant information is given by the previous blowing-up. Glueing the different charts together we obtain the phase-portrait given in Figure 2.7 (a). Taking into account (8) we obtain the topological types of Figure 2.7 (b) for $X_{R}$ which depend on the signs of $a_{1}$ and $b_{4}$.

(a)

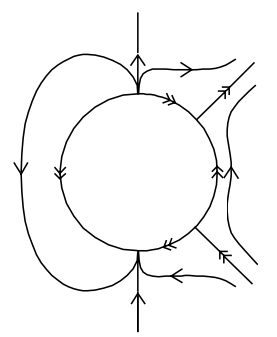

(b)

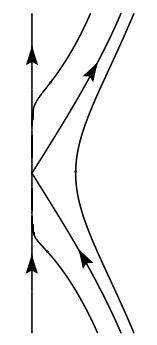

$$
\begin{array}{cc}
\left(a_{1}, b_{4}\right)=(1,1) & \left(a_{1}, b_{4}\right)=(-1,-1) \\
a_{1}>0, b_{4}>0 & a_{1}<0, b_{4}<0
\end{array}
$$

Figure 2.7. Topological types for vector fields in $N_{4,2} \backslash N_{5,4}$ with $a_{1} b_{4}>0$. We use the model $\frac{a_{1}}{2} x y \frac{\partial}{\partial x}+\left(x^{2}+b_{4} y^{4}\right) \frac{\partial}{\partial y}$. 
On the other hand, when $a_{1} b_{4}<0$ we use the change of variables and the rescaling given by

$$
\left\{\begin{array}{l}
x=-\frac{a_{1}^{2}}{b_{4}} \bar{x} \\
y=\left(-\frac{a_{1}}{b_{4}}\right)^{\frac{1}{2}} \bar{y}
\end{array} t=-\left(-\frac{b_{4}}{a_{1}}\right)^{\frac{3}{2}} \frac{1}{b_{4}} \bar{t}\right.
$$

to reduce $(5)$ to

$$
\left\{\begin{array}{l}
\bar{x}^{\prime}=\bar{x} \bar{y}+\bar{x}(o(\bar{y})) \\
\bar{y}^{\prime}=\bar{x}-\bar{y}^{4}+o\left(\bar{y}^{4}\right) .
\end{array}\right.
$$

In this case, by blowing-up in the $\bar{y}$-direction with $v=1$, we get

$$
\left\{\begin{array}{l}
r^{\prime}=r u-r^{3}+o\left(r^{3}\right) \\
u^{\prime}=u-2 u^{2}+u(O(r)) .
\end{array}\right.
$$

As in the previous cases the above vector field gives us all the relevant information. We obtain the phase-portrait given in Figure 2.8 (a) and the topological types given in Figure 2.8 (b) taking into account the signs of $a_{1}$ and $b_{4}$ in $(9)$.

(a)

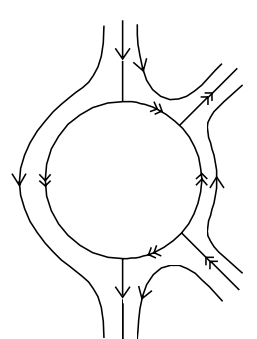

(b)

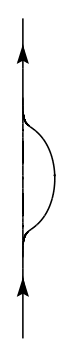

$$
\begin{array}{cc}
\left(a_{1}, b_{4}\right)=(-1,1) & \left(a_{1}, b_{4}\right)=(1,-1) \\
a_{1}<0, b_{4}>0 & a_{1}>0, b_{4}<0
\end{array}
$$

Figure 2.8. Topological types for vector fields in $N_{4,2} \backslash N_{5,4}$ with $a_{1} b_{4}<0$. We use the model $\frac{a_{1}}{2} x y \frac{\partial}{\partial x}+\left(x^{2}+b_{4} y^{4}\right) \frac{\partial}{\partial y}$.

Case 3: Now we use a different blowing-up given by

$$
\bar{x}=r^{3} u, \quad \bar{y}=r v .
$$


Blowing-up in the $\bar{y}$-direction with $v=1$ we obtain, after division by $r^{2}$,

$$
\left\{\begin{array}{l}
r^{\prime}=r u+b_{3} r+o(r) \\
u^{\prime}=a_{2} u-3 u\left(u+b_{3}\right)+O(r) .
\end{array}\right.
$$
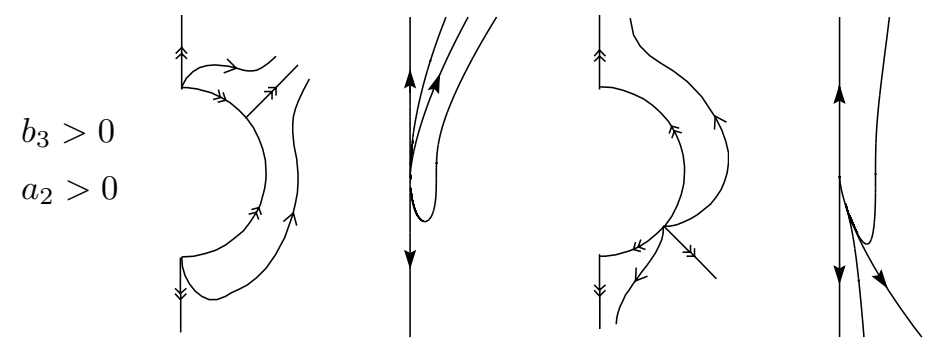

$$
\left(a_{2}, b_{3}\right)=(4,1)
$$

$\left(a_{2}, b_{3}\right)=(1,1)$
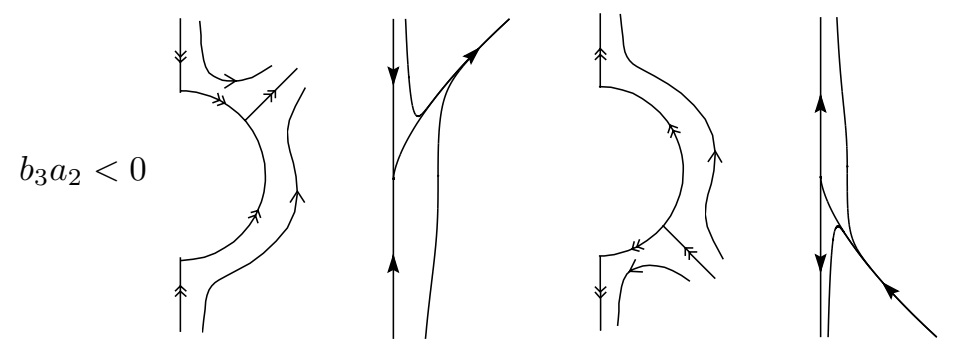

$$
\left(a_{2}, b_{3}\right)=(1,-1)
$$

$\left(a_{2}, b_{3}\right)=(-1,1)$
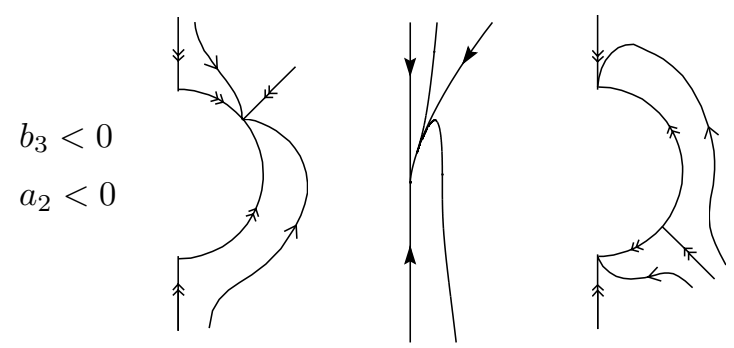

$$
\begin{gathered}
\left(a_{2}, b_{3}\right)=(-1,-1) \\
a_{2}-3 b_{3}>0
\end{gathered}
$$

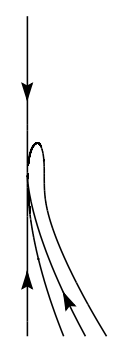

$$
\begin{gathered}
\left(a_{2}, b_{3}\right)=(-4,-1) \\
a_{2}-3 b_{3}<0
\end{gathered}
$$

Figure 2.9. Topological types for vector fields in $N_{4,1} \backslash\left(N_{5,1} \cup N_{5,2} \cup N_{5,3}\right)$. We use the model $\frac{a_{2}}{2} x y^{2} \frac{\partial}{\partial x}+\left(x^{2}+b_{3} y^{3}\right) \frac{\partial}{\partial y}$. 
We find exactly two hyperbolic singularities at $(r, u)=(0,0)$ and $(r, u)=$ $\left(0,\left(a_{2}-3 b_{3}\right) / 3\right)$. The blowing-up in the $y$-direction with $v=-1$ is related to the one obtained by taking $v=1$ by means of the change $(r, u) \rightarrow$ $(-r,-u)$. All the relevant information is given by the previous vector field and glueing the different charts together we get the phase-portraits given in Figure 2.9 for the blowing-up near $S^{1} \times\{0\}$ as well as for the different topological types corresponding to $X_{R}$.

\section{Quadratic case $\left(b_{01}=0\right)$}

Under certain conditions we will consider further simplifications of the expresion of $X_{R}$ given in (4). These simplifications are obtained by means of linear changes of coordinates and they are such that the 2-jet of (4) with respect to the new coordinates presents some specific invariant lines. Let us recall that the 2-jet of $X_{R}$ is given by

$$
x\left(a_{10} y+a_{01} x\right) \frac{\partial}{\partial x}+\left(b_{20} y^{2}+b_{11} y x+b_{02} x^{2}\right) \frac{\partial}{\partial y} .
$$

Simple calculations permit to show that if $A$ is such that

$$
\left(a_{10}-b_{20}\right) A^{2}+\left(a_{01}-b_{11}\right) A-b_{02}=0,
$$

then the line $y=A x$ is invariant for the vector field given in (10). Let

$$
\Delta=\left(a_{01}-b_{11}\right)^{2}+4 b_{02}\left(a_{10}-b_{20}\right)
$$

be the discriminant of equation (11).

If $\Delta<0$ then $a_{10}-b_{20} \neq 0$ and hence (11) is a quadratic equation which has no real solutions. In this case no further simplifications are done and we merely define the following strata in $W_{2}$ :

$$
\begin{aligned}
& C_{3,1}=\left\{X \in W_{2} \mid b_{01}=0, \Delta<0\right\}, \\
& C_{4,1}=\left\{X \in C_{3,1} \mid b_{20}=0\right\}, \\
& C_{5,1}=\left\{X \in C_{3,1} \mid b_{20}=0, b_{30}=0\right\} .
\end{aligned}
$$


If $\Delta>0$ and $a_{10}-b_{20} \neq 0$ then (11) is a quadratic equation with two different real solutions $A_{1}$ and $A_{2}$ for which condition $A_{2}>A_{1}$ is assumed. The linear change of coordinates

$$
\left\{\begin{array}{l}
x=\bar{x} \\
y=A_{1} \bar{x}+\left(A_{2}-A_{1}\right) \bar{y}
\end{array}\right.
$$

sends the lines $\left\{y=A_{1} x\right\}$ and $\left\{y=A_{2} x\right\}$ to $\{\bar{y}=0\}$ and $\{\bar{y}=\bar{x}\}$, respectively. Hence, with respect to the $(\bar{x}, \bar{y})$-coordinates, $X_{R}$ still maintains $\{\bar{x}=0\}$ as an invariant line and presents the following 3 -jet:

(13) $\bar{x}\left(\bar{a}_{10} \bar{y}+\bar{a}_{01} \bar{x}+\bar{a}_{20} \bar{y}^{2}+\bar{a}_{11} \bar{y} \bar{x}+\bar{a}_{02} \bar{x}^{2}\right) \frac{\partial}{\partial \bar{x}}$

$$
+\left(\bar{b}_{20} \bar{y}^{2}+\bar{b}_{11} \bar{y} \bar{x}+\bar{b}_{30} \bar{y}^{3}+\bar{b}_{21} \bar{y}^{2} \bar{x}+\bar{b}_{12} \bar{y} \bar{x}^{2}+\bar{b}_{03} \bar{x}^{3}\right) \frac{\partial}{\partial \bar{y}}
$$

with $\bar{b}_{20}+\bar{b}_{11}=\bar{a}_{01}+\bar{a}_{10}$ since $\bar{y}=\bar{x}$ is an invariant line for the 2-jet. Under these conditions we define the following stratification:

$$
\begin{aligned}
C_{3,2}= & \left\{X \in W_{2} \mid b_{01}=0, \Delta>0, a_{10}-b_{20} \neq 0\right\}, \\
C_{4,2}= & \left\{X \in C_{3,2} \mid \bar{b}_{20}=0\right\}, \\
C_{4,3}= & \left\{X \in C_{3,2} \mid \bar{b}_{20} \neq 0, \bar{a}_{01}=0\right\}, \\
C_{4,4}= & \left\{X \in C_{3,2} \mid \bar{b}_{20} \neq 0, \bar{a}_{01} \neq 0, \bar{a}_{01}+\bar{a}_{10}=0\right\}, \\
C_{5,2}= & \left\{X \in C_{3,2} \mid \bar{b}_{20}=0, \bar{a}_{01}=0\right\}, \\
C_{5,3}= & \left\{X \in C_{3,2} \mid \bar{b}_{20}=0, \bar{a}_{01} \neq 0, \bar{a}_{01}+\bar{a}_{10}=0\right\}, \\
C_{5,4}= & \left\{X \in C_{3,2} \mid \bar{b}_{20}=0, \bar{a}_{01} \neq 0, \bar{a}_{01}+\bar{a}_{10} \neq 0, \bar{b}_{30}=0\right\}, \\
C_{5,5}= & \left\{X \in C_{3,2} \mid \bar{b}_{20} \neq 0, \bar{a}_{01}=0, \bar{a}_{10}=0\right\}, \\
C_{5,6}= & \left\{X \in C_{3,2} \mid \bar{b}_{20} \neq 0, \bar{a}_{01}=0, \bar{a}_{10} \neq 0, \bar{a}_{10} \bar{b}_{03}+\bar{a}_{02}\left(\bar{b}_{20}-\bar{a}_{10}\right)=0\right\}, \\
C_{5,7}= & \left\{X \in C_{3,2} \mid \bar{b}_{20} \neq 0, \bar{a}_{01} \neq 0, \bar{a}_{01}+\bar{a}_{10}=0, \bar{b}_{20}\left(\bar{a}_{20}+\bar{a}_{11}+\bar{a}_{02}\right)\right. \\
& \left.-\bar{a}_{10}\left(\bar{b}_{30}+\bar{b}_{21}+\bar{b}_{12}+\bar{b}_{03}\right)=0\right\} .
\end{aligned}
$$


Finally, we have to consider the cases $\left\{\Delta>0, a_{10}-b_{20}=0\right\}$ and $\left\{\Delta=0, a_{10}-b_{20} \neq 0\right\}$. The condition in the first case implies that $a_{01}-b_{11} \neq 0$ and therefore (11) is a linear equation. In the second case (11) is a second order equation with a unique real solution. Let $A_{1}$ be the unique solution of (11) under any of the previous conditions. In such a case, with respect to the $(\bar{x}, \bar{y})$-coordinates given by the linear transformation

$$
\begin{aligned}
& x=\bar{x} \\
& y=A_{1} \bar{x}+\bar{y},
\end{aligned}
$$

the vector field $X_{R}$ still maintains $\{\bar{x}=0\}$ as an invariant line and its 3 -jet is like in (13). That is, the 2 -jet presents $\{\bar{y}=0\}$ as the unique invariant line of the type $\bar{y}=A \bar{x}$. Thus, we define the following strata of codimension 4 and 5 :

$$
\begin{aligned}
& C_{4,5}=\left\{X \in W_{2} \mid b_{01}=0, \Delta>0, a_{10}-b_{20}=0\right\}, \\
& C_{5,8}=\left\{X \in C_{4,5} \mid \bar{b}_{20}=0\right\}, \\
& C_{5,9}=\left\{X \in C_{4,5} \mid \bar{b}_{20} \neq 0, \bar{a}_{01}=0\right\}
\end{aligned}
$$

and

$$
\begin{aligned}
C_{4,6} & =\left\{X \in W_{2} \mid b_{01}=0, \Delta=0, a_{10}-b_{20} \neq 0\right\}, \\
C_{5,10} & =\left\{X \in C_{4,6} \mid \bar{b}_{20}=0\right\}, \\
C_{5,11} & =\left\{X \in C_{4,6} \mid \bar{b}_{20} \neq 0, \bar{a}_{01}=0\right\} .
\end{aligned}
$$

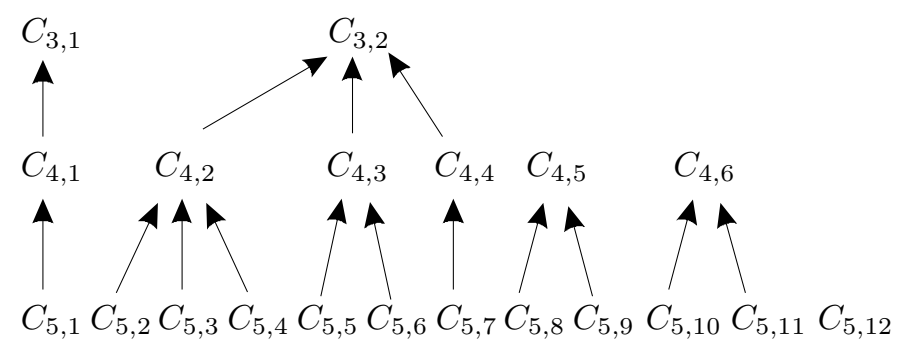

Figure 3.1. Stratification in the quadratic case. 
We also define the stratum $C_{5,12}=\left\{X \in W_{2} \mid b_{01}=0, \Delta=0\right.$, $\left.a_{10}-b_{20}=0\right\}$. Note that each $C_{i, j}$ with $i=3,4,5$ is a semi-algebraic set of codimension $i$. In Figure 3.1 we show how the different strata are organized.

Remark 3.1. The $a_{i j}$ and $b_{i j}$ refer to coefficients in (4) while $\bar{a}_{i j}$ and $\bar{b}_{i j}$ to coefficients in (13). Nevertheless, it is important to observe that after the change (12) (resp. (14)) $\bar{a}_{10}=a_{10}\left(A_{2}-A_{1}\right)$ and $\bar{b}_{20}=b_{20}\left(A_{2}-A_{1}\right)\left(\right.$ resp. $\bar{a}_{10}=a_{10}$ and $\left.\bar{b}_{20}=b_{20}\right)$. Therefore, in both cases $\bar{a}_{10}-\bar{b}_{20}$ and $a_{10}-b_{20}$ have the same sign. Since $\bar{y}=0$ is the unique invariant line of the type $\bar{y}=A \bar{x}$ for the 2-jet of (13) if $X \in C_{4,5}$, it follows that $A=0$ is the unique solution of $\left(\bar{a}_{01}-\bar{b}_{11}\right) A=0$ and hence $\bar{a}_{01}-\bar{b}_{11} \neq 0$. Similarly, if $X \in C_{4,6}$ then $A=0$ is the unique solution of $\left(\bar{a}_{10}-\bar{b}_{20}\right) A^{2}+\left(\bar{a}_{01}-\bar{b}_{11}\right) A=0$ and hence $\bar{a}_{01}=\bar{b}_{11}$.

Remark 3.2. Putting together the different strata which we have just characterized with the ones given for the nilpotent case in section 2, we finally obtain the following stratification of $W_{2}$ :

$$
\begin{aligned}
& W_{22}=N_{2,1} \\
& W_{23}=\left(\cup_{j=1}^{3} N_{3, j}\right) \cup\left(\cup_{j=1}^{2} C_{3, j}\right), \\
& W_{24}=\left(\cup_{j=1}^{3} N_{4, j}\right) \cup\left(\cup_{j=1}^{6} C_{4, j}\right), \\
& W_{25}=\left(\cup_{j=1}^{5} N_{5, j}\right) \cup\left(\cup_{j=1}^{12} C_{5, j}\right) .
\end{aligned}
$$

Note that each $W_{2 i}$ with $i=2,3,4,5$ is a semi-algebraic set of codimension $i$.

We will obtain the different equivalence classes which are found in each stratum $C_{i, j} \backslash C_{i, j+1}$. To do this we use the homogeneous blow-up given by

$$
x=r u, \quad y=r v .
$$

Since the exponent of $r$ in the change $y=r v$ is odd the vector field obtained by blowing-up in the $y$-direction with $v=-1$ is related to the one obtained by taking $v=1$ by means of the change $(r, u) \rightarrow(-r,-u)$ and $t \rightarrow-t$. We will keep this in mind in the sequel. 
We can first give a survey of the different dynamics which are observed in each stratum as well as of the different transitions. We first study the singularities obtained after one blow-up. Only the side $u \geq 0$ on the blown-up circle needs to be considered. In all cases we will find two singularities at $(u, v)=(0, \pm 1)$, corresponding to the invariant axes. For $C_{3,1}$ there are no more singularities and further degeneracies in $C_{4,1}$ and $C_{5,1}$ are given by the coalescence of an extra hyperbolic singular point with $(0, \pm 1)$. For $C_{3,2}$ we also find two singularities $p_{1}$ and $p_{2}$ on the blown-up circle with $u>0$. Further degeneracies along the different strata contained in $C_{3,2}$ are given by the coalescence of an extra hyperbolic singular point with $(0, \pm 1), p_{1}$ or $p_{2} . C_{4,5}$ can be seen as the transition inside $C_{3,2}$ from $a_{10}-b_{20}$ positive to negative. As a consequence, one of the singularities, either $p_{1}$ or $p_{2}$, coalesce with $(0, \pm 1)$ in a saddle-node bifurcation. Finally $C_{4,6}$ shows the transition from $C_{3,2}$ to $C_{3,1}$, in which $p_{1}$ and $p_{2}$ coalesce in a saddle-node bifurcation.

Remark 3.3. Let us recall that in the previous calculations on (3) the coordinates $(x, y)$ represent in fact $(w, z)$ in expression $(2)$. Going back to the original coordinates $(x, y)$ in (1) we have to use $w=x^{2}$ and $z=y$. By means of blow-up we prove the existence of invariant curves for vector fields like in (3) of the form $z=\alpha w+o(w)$. Hence, with respect to $(x, y)$-coordinates we get invariant curves of type $y=\alpha x^{2} / 2+o\left(x^{2}\right)$. This must be kept in mind when drawing, as we do in this case, the different topological types for vector fields (1).

\subsection{Singularities of codimension 3 .}

We will distinguish two cases:

1. $X \in C_{3,1} \backslash C_{4,1}$. In this case $b_{01}=0, \Delta<0$ and $b_{20} \neq 0$,

2. $X \in C_{3,2} \backslash\left(C_{4,2} \cup C_{4,3} \cup C_{4,4}\right)$. In this case $b_{01}=0, \Delta>0$, $a_{10}-b_{20} \neq 0, \bar{b}_{20} \neq 0, \bar{a}_{01} \neq 0$ and $\bar{a}_{01}+\bar{a}_{10} \neq 0$,

which are studied separately.

Case 1: We consider expression (4) for $X_{R}$. The change of coordinates $(x, y) \rightarrow(x,-y)$ permits us to put $b_{20}>0$. Hence, we assume that $b_{20}$ is positive. Blowing-up in the $y$-direction with $v=1$ we get

$$
\left\{\begin{array}{l}
r^{\prime}=b_{20} r+r(O(\|(r, u)\|)) \\
u^{\prime}=u\left(a_{10}-b_{20}+\left(a_{01}-b_{11}\right) u-b_{02} u^{2}\right)+u(O(r)) .
\end{array}\right.
$$


Since $\Delta<0$, on the invariant line $\{r=0\}$ we find exactly one hyperbolic singularity at $(r, u)=(0,0)$. Recall that $\Delta<0$ implies that $\left(a_{10}-b_{20}\right) \neq 0$. One can check that blowing-up in the $x$-direction no singularity is found at $(r, v)=(0,0)$. The study of the singularity leads to the phase-portraits given in Figure 3.2 for the blowing-up as well as for the corresponding topological types of $X_{R}$.

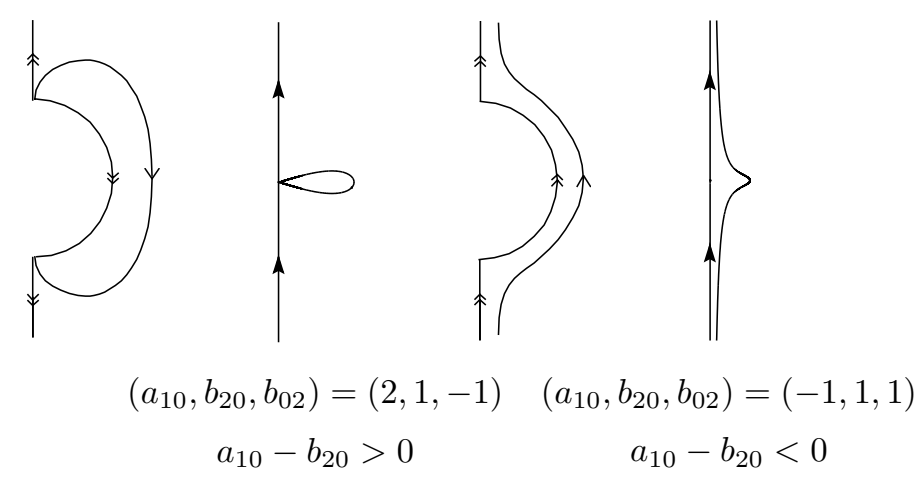

Figure 3.2. Topological types for vector fields in $C_{3,1} \backslash C_{4,1}$.

We use the model $\frac{1}{2} x\left(a_{10} y+a_{01} x^{2}\right) \frac{\partial}{\partial x}+\left(b_{20} y^{2}+b_{11} y x^{2}+\right.$ $\left.b_{02} x^{4}\right) \frac{\partial}{\partial y}$.

Case 2: In this case we consider the expression of $X_{R}$ which is obtained after the change (12) and hence we assume that its 3 -jet is given by (13). As it is explained in Remark 3.1, $\bar{a}_{10}-\bar{b}_{20} \neq 0$. Moreover we use that $\bar{b}_{20}+\bar{b}_{11}=\bar{a}_{01}+\bar{a}_{10}$ and assume, as in the previous case, that $\bar{b}_{20}>0$.

Blowing-up in the $\bar{x}$-direction with $u=1$ we get

$$
\left\{\begin{array}{l}
r^{\prime}=\left(\bar{a}_{10} v+\bar{a}_{01}\right) r+o(r) \\
v^{\prime}=\left(\bar{b}_{20}-\bar{a}_{10}\right) v(v-1)+O(r) .
\end{array}\right.
$$

On the invariant line $\{r=0\}$ we find exactly two hyperbolic singularities at $(r, u)=(0,0)$ and $(r, v)=(0,1)$. Blowing-up in the $\bar{y}$-direction with $v=1$ we get an extra hyperbolic singularity at $(r, u)=(0,0)$. Studying the singularities and glueing the different charts together we obtain the phase-portraits given in Figure 3.3. 


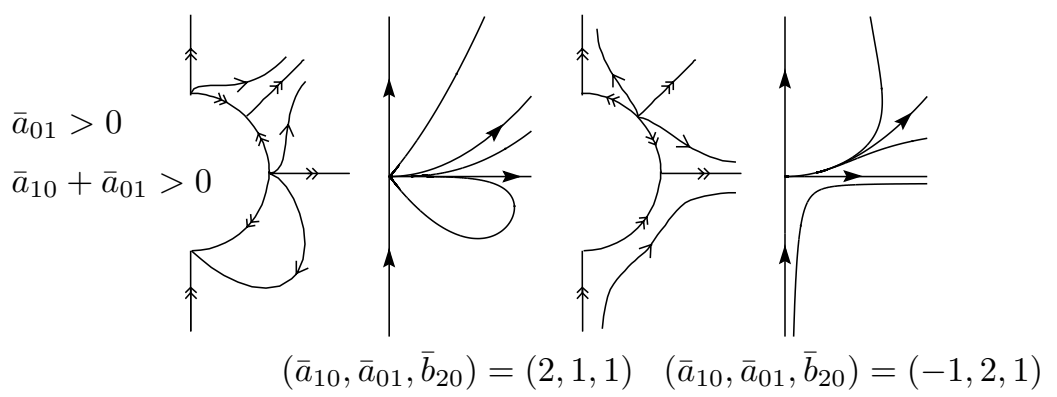

$$
\begin{aligned}
& \bar{a}_{01}>0 \\
& \bar{a}_{10}+\bar{a}_{01}<0
\end{aligned}
$$

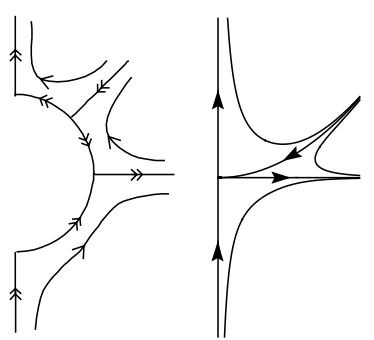

$$
\left(\bar{a}_{10}, \bar{a}_{01}, \bar{b}_{20}\right)=(-2,1,1)
$$

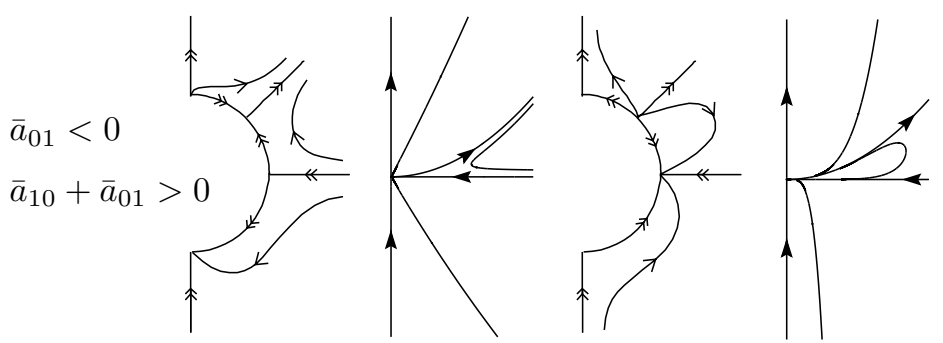

$$
\left(\bar{a}_{10}, \bar{a}_{01}, \bar{b}_{20}\right)=(2,-1,1)\left(\bar{a}_{10}, \bar{a}_{01}, \bar{b}_{20}\right)=(1 / 2,-1 / 4,1)
$$

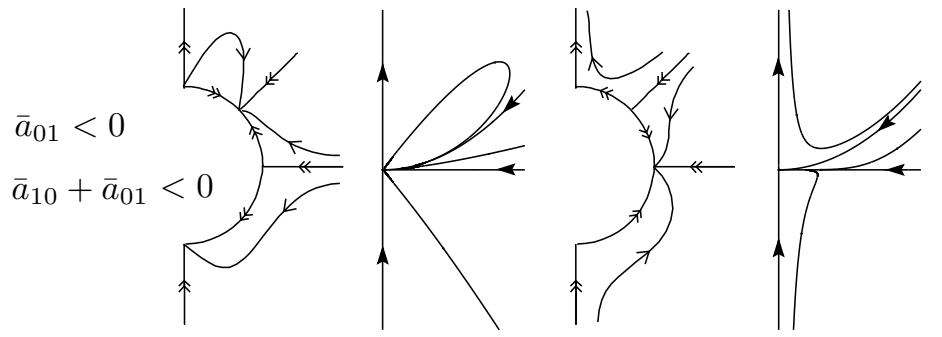

$$
\begin{array}{cc}
\left(\bar{a}_{10}, \bar{a}_{01}, \bar{b}_{20}\right)=(2,-3,1) & \left(\bar{a}_{10}, \bar{a}_{01}, \bar{b}_{20}\right)=(-1,-1,1) \\
\bar{a}_{10}-\bar{b}_{20}>0 & \bar{a}_{10}-\bar{b}_{20}<0
\end{array}
$$

Figure 3.3. Topological types for vector fields in $C_{3,2} \backslash\left(C_{4,2} \cup C_{4,3} \cup C_{4,4}\right)$. We use the model $\frac{1}{2} x\left(\bar{a}_{10} y+\bar{a}_{01} x^{2}\right) \frac{\partial}{\partial x}+\left(\bar{b}_{20} y^{2}+\left(\bar{a}_{01}+\bar{a}_{10}-\right.\right.$ $\left.\left.\bar{b}_{20}\right) y x^{2}\right) \frac{\partial}{\partial y}$. 


\subsection{Singularities of codimension 4 .}

We have six cases:

1. $X \in C_{4,1} \backslash C_{5,1}$. In this case $b_{01}=0, \Delta<0, b_{20}=0$ and $b_{30} \neq 0$,

2. $X \in C_{4,2} \backslash\left(C_{5,2} \cup C_{5,3} \cup C_{5,4}\right)$. In this case $b_{01}=0, \Delta>0$, $a_{10}-b_{20} \neq 0, \bar{b}_{20}=0, \bar{a}_{01} \neq 0, \bar{a}_{01}+\bar{a}_{10} \neq 0$ and $b_{30} \neq 0$,

3. $X \in C_{4,3} \backslash\left(C_{5,5} \cup C_{5,6}\right)$. In this case $b_{01}=0, \Delta>0, a_{10}-b_{20} \neq 0$, $\bar{b}_{20} \neq 0, \bar{a}_{01}=0, \bar{a}_{10} \neq 0$ and $\bar{a}_{10} \bar{b}_{03}+\bar{a}_{02}\left(\bar{b}_{20}-\bar{a}_{10}\right) \neq 0$,

4. $X \in C_{4,4} \backslash C_{5,7}$. In this case $b_{01}=0, \Delta>0, a_{10}-b_{20} \neq 0$, $\bar{b}_{20} \neq 0, \bar{a}_{01} \neq 0, \bar{a}_{01}+\bar{a}_{10}=0$ and $\bar{b}_{20}\left(\bar{a}_{20}+\bar{a}_{11}+\bar{a}_{02}\right)-\bar{a}_{10}\left(\bar{b}_{30}+\right.$ $\left.\bar{b}_{21}+\bar{b}_{12}+\bar{b}_{03}\right) \neq 0$,

5. $X \in C_{4,5} \backslash\left(C_{5,8} \cup C_{5,9}\right)$. In this case $b_{01}=0, \Delta>0, a_{10}-b_{20}=0$, $\bar{b}_{20} \neq 0$ and $\bar{a}_{01} \neq 0$,

6. $X \in C_{4,6} \backslash\left(C_{5,10} \cup C_{5,11}\right)$. In this case $b_{01}=0, \Delta=0, a_{10}-b_{20} \neq 0$, $\bar{b}_{20} \neq 0$ and $\bar{a}_{01} \neq 0$,

which are studied separately.

Case 1: In this case the change of coordinates $(\bar{x}, \bar{y}) \rightarrow(\bar{x},-\bar{y})$ permit us to put $a_{10}>0$ and hence we assume that such a condition is satisfied. Blowing-up (4) in the $y$-direction with $v=1$ we get

$$
\left\{\begin{array}{l}
r^{\prime}=r\left(b_{11} u+b_{02} u^{2}\right)+r^{2}\left(b_{30}+b_{21} u+b_{12} u^{2}+b_{03} u^{3}\right)+o\left(r^{2}\right) \\
u^{\prime}=u\left(a_{10}+\left(a_{01}-b_{11}\right) u-b_{02} u^{2}\right)+u(O(r))
\end{array}\right.
$$
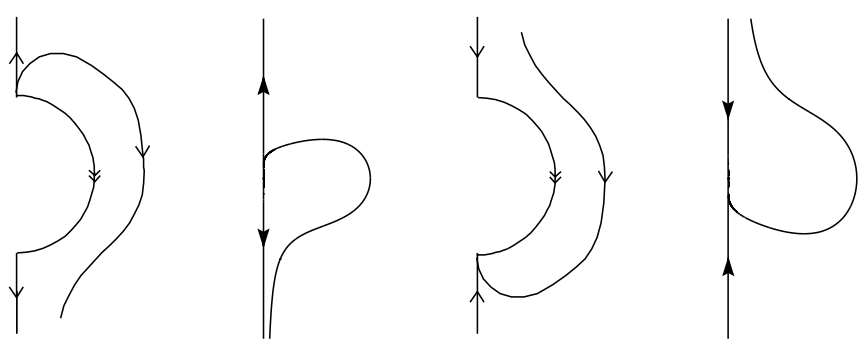

$$
\begin{aligned}
\left(a_{10}, b_{02}, b_{30}\right) & =(1,-1,1) & \left(a_{10}, b_{02}, b_{30}\right) & =(1,-1,-1) \\
b_{30} & >0 & b_{30} & <0
\end{aligned}
$$

Figure 3.4. Topological types for vector fields in $C_{4,1} \backslash C_{5,1}$.

We use the model $\frac{1}{2} x\left(a_{10} y+a_{01} x^{2}\right) \frac{\partial}{\partial x}+\left(b_{11} y x^{2}+b_{02} x^{4}+\right.$ $\left.b_{30} y^{3}\right) \frac{\partial}{\partial y}$. 
Since $\Delta<0$, on the invariant line $\{r=0\}$ we find exactly one singularity at $(r, u)=(0,0)$, where the linear part is given by $a_{10} u \frac{\partial}{\partial u}$. Therefore, there exists a center manifold tangent to the eigenspace associated to the 0 eigenvalue. In this case $\{u=0\}$ is an invariant line and hence it is a center manifold. The restriction of the vector field to $\{u=0\}$ is given by $\left(b_{30} r^{2}+o\left(r^{2}\right)\right) \frac{\partial}{\partial r}$. One can check that blowing-up in the $x$-direction no singularity is found at $(r, v)=(0,0)$. Glueing the different charts together we get the phase-portraits given in Figure 3.4 for the blowingup near $S^{1} \times\{0\}$ as well as for the corresponding topological types of $X_{R}$.

Case 2: We use the expression of the 3-jet of $X_{R}$ which is given in (13) and, similarly to the previous case, we may assume that $\bar{a}_{10}>0$. By blowing-up $X_{R}$ in the $\bar{x}$-direction we will obtain the same phaseportraits as in case 2 of codimension 3 . Nevertheless, blowing-up in the $\bar{y}$-direction with $v=1$ we get

$$
\left\{\begin{array}{l}
r^{\prime}=\bar{b}_{11} r u+r^{2}\left(\bar{b}_{30}+\bar{b}_{21} u+\bar{b}_{12} u^{2}+\bar{b}_{03} u^{3}\right)+o\left(r^{2}\right) \\
u^{\prime}=u\left(\bar{a}_{10}+\left(\bar{a}_{01}-\bar{b}_{11}\right) u\right)+u(O(r)) .
\end{array}\right.
$$

The linear part at $(r, u)=(0,0)$ is given by $\bar{a}_{10} u \frac{\partial}{\partial u}$. As in the previous case, $\{u=0\}$ is a center manifold. The restriction of the vector field to $\{u=0\}$ is given by $\left(\bar{b}_{30} r^{2}+o\left(r^{2}\right)\right) \frac{\partial}{\partial r}$. In Figure 3.5 the corresponding phase-portraits for the blowing-up and for $X_{R}$ are depicted.

Case 3: We assume that $\bar{b}_{20}>0$. After blowing-up we obtain the same singularities as in case 2 of codimension 3 . Also the behaviour at each one of them is the same except for the singularity at $(r, v)=(0,0)$ which is obtained by blowing-up in the $\bar{x}$-direction with $u=1$. In such a case we get the vector field

$$
\left\{\begin{aligned}
r^{\prime}= & \bar{a}_{10} r v+r^{2}\left(\bar{a}_{20} v^{2}+\bar{a}_{11} v+\bar{a}_{02}\right)+o\left(r^{2}\right) \\
v^{\prime}= & \left(\bar{b}_{20}-\bar{a}_{10}\right) v(v-1)+r\left(\left(\bar{b}_{30}-\bar{a}_{20}\right) v^{3}\right. \\
& \left.\quad+\left(\bar{b}_{21}-\bar{a}_{11}\right) v^{2}+\left(\bar{b}_{12}-\bar{a}_{02}\right) v+\bar{b}_{03}\right)+o(r) .
\end{aligned}\right.
$$

The linear part at $(r, v)=(0,0)$ is given by $\left(\bar{b}_{03} r+\left(\bar{a}_{10}-\bar{b}_{20}\right) v\right) \frac{\partial}{\partial v}$. The singularity is hyperbolic for the restriction to $\{r=0\}$ and there exists a center manifold $W^{c}$ tangent to the eigenspace associated to the 0 eigenvalue. A simple calculation shows that a first order approximation of $W^{c}$ is given by

$$
\frac{\bar{b}_{03}}{\bar{b}_{20}-\bar{a}_{10}} r+o(r)
$$


and the reduction of the vector field to $W^{c}$ is

$$
r^{\prime}=\frac{\bar{a}_{10} \bar{b}_{03}+\bar{a}_{02}\left(\bar{b}_{20}-\bar{a}_{10}\right)}{\bar{b}_{20}-\bar{a}_{10}} r^{2}+o\left(r^{2}\right) .
$$
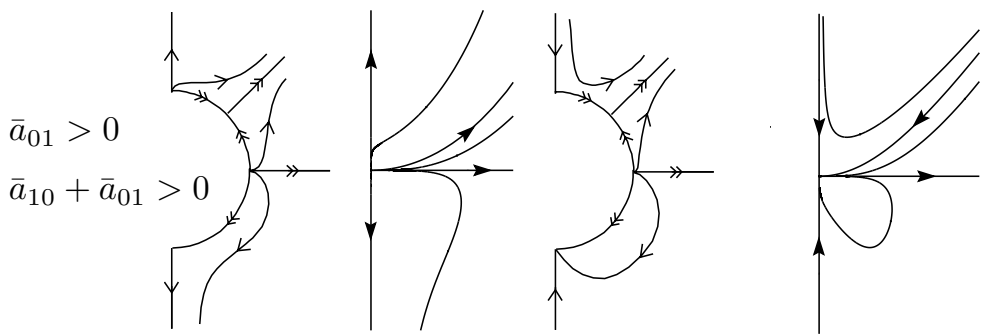

$$
\left(\bar{a}_{10}, \bar{a}_{01}, \bar{b}_{30}\right)=(1,1,1) \quad\left(\bar{a}_{10}, \bar{a}_{01}, \bar{b}_{30}\right)=(1,1,-1)
$$

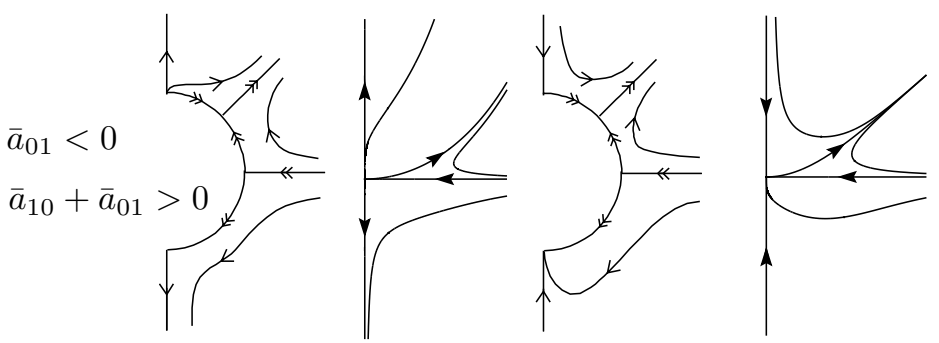

$\left(\bar{a}_{10}, \bar{a}_{01}, \bar{b}_{30}\right)=(1,-1 / 2,1) \quad\left(\bar{a}_{10}, \bar{a}_{01}, \bar{b}_{30}\right)=(1,-1 / 2,-1)$
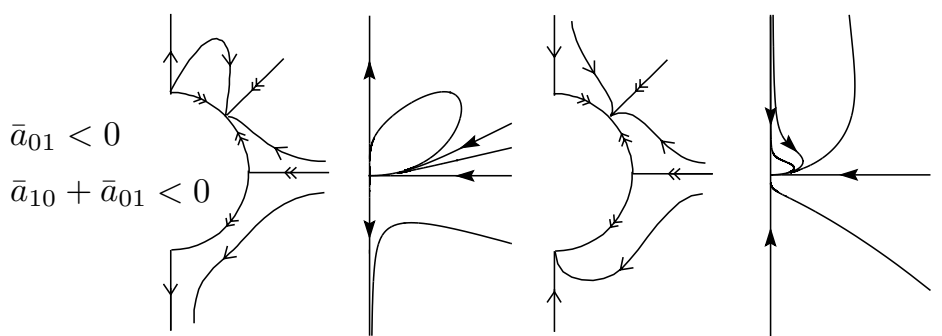

$$
\begin{array}{rlrl}
\left(\bar{a}_{10}, \bar{a}_{01}, \bar{b}_{30}\right)=(1,-2,1) & \left(\bar{a}_{10}, \bar{a}_{01}, \bar{b}_{30}\right) & =(1,-2,-1) \\
\bar{b}_{30} & >0 & \bar{b}_{30} & <0
\end{array}
$$

Figure 3.5. Topological types for vector fields in $C_{4,2} \backslash\left(C_{5,2} \cup C_{5,3} \cup C_{5,4}\right)$. We use the model $\frac{1}{2} x\left(\bar{a}_{10} y+\bar{a}_{01} x^{2}\right) \frac{\partial}{\partial x}+\left(\left(\bar{a}_{01}+\bar{a}_{10}\right) y x^{2}+\right.$ $\left.\bar{b}_{30} y^{3}\right) \frac{\partial}{\partial y}$. 
We get the phase-portraits depicted in Figure 3.6 for the blowing-up and for the topological types of $X_{R}$.

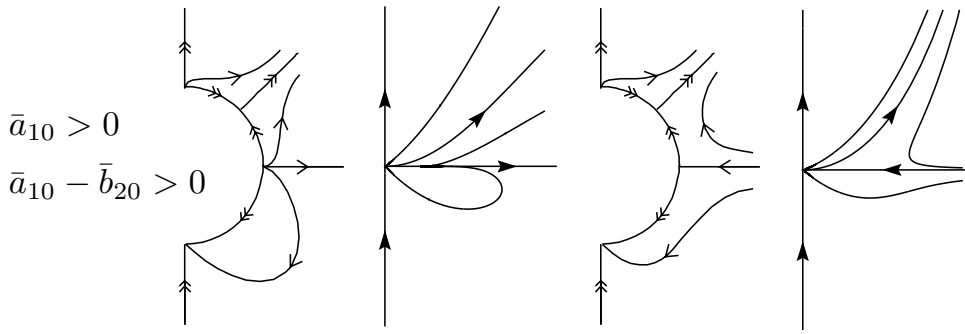

$\left(\bar{a}_{10}, \bar{a}_{02}, \bar{b}_{20}\right)=(2,1,1) \quad\left(\bar{a}_{10}, \bar{a}_{02}, \bar{b}_{20}\right)=(2,-1,1)$
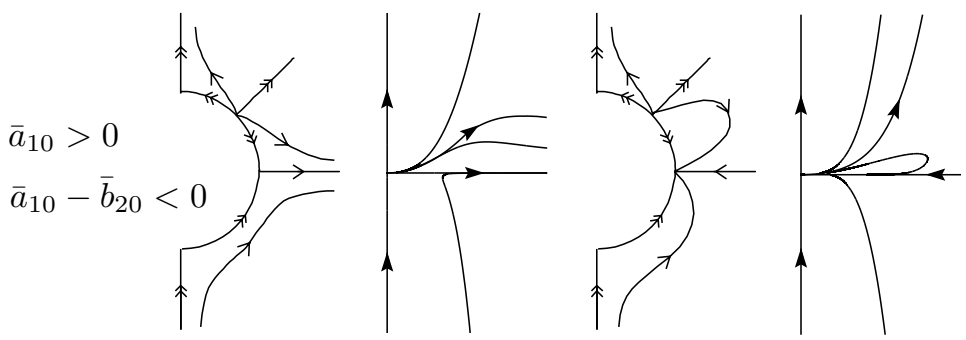

$\left(\bar{a}_{10}, \bar{a}_{02}, \bar{b}_{20}\right)=(1 / 2,1,1)\left(\bar{a}_{10}, \bar{a}_{02}, \bar{b}_{20}\right)=(1 / 2,-1,1)$
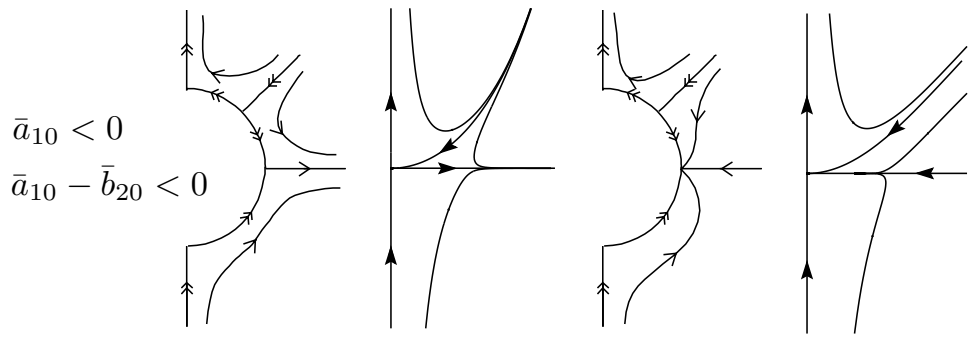

$$
\begin{array}{cc}
\left(\bar{a}_{10}, \bar{a}_{02}, \bar{b}_{20}\right)=(-1,1,1) & \left(\bar{a}_{10}, \bar{a}_{02}, \bar{b}_{20}\right)=(-1,-1,1) \\
\alpha>0 & \alpha<0
\end{array}
$$

Figure 3.6. Topological types for vector fields in $C_{4,3} \backslash\left(C_{5,5} \cup C_{5,6}\right)$. $\alpha=\left(\bar{a}_{10} \bar{b}_{03}+\bar{a}_{02}\left(\bar{b}_{20}-\bar{a}_{10}\right)\right) /\left(\bar{b}_{20}-\bar{a}_{10}\right)$. We use the model $\frac{1}{2} x\left(\bar{a}_{10} y+\bar{a}_{20} y^{2}+\bar{a}_{02} x^{4}\right) \frac{\partial}{\partial x}+\left(\bar{b}_{20} y^{2}+\left(\bar{a}_{10}-\bar{b}_{20}\right) y x^{2}+\bar{b}_{03} x^{6}\right) \frac{\partial}{\partial y}$. 
Case 4: Again, we assume that $\bar{b}_{20}>0$. As in the previous case, after blowing-up, we obtain the same singularities that in case 2 of codimension 3. The behaviour at each one of them is the same except for the singularity which is present at $(r, v)=(0,1)$ after blowing-up in the $\bar{x}$-direction with $u=1$. In such a case we get the vector field

$$
\left\{\begin{aligned}
r^{\prime}= & r\left(\bar{a}_{10} v+\bar{a}_{01}\right)+r^{2}\left(\bar{a}_{20} v^{2}+\bar{a}_{11} v+\bar{a}_{02}\right)+o\left(r^{2}\right) \\
v^{\prime}= & \left(\bar{b}_{20}-\bar{a}_{10}\right) v(v-1)+r\left(\left(\bar{b}_{30}-\bar{a}_{20}\right) v^{3}\right. \\
& \left.+\left(\bar{b}_{21}-\bar{a}_{11}\right) v^{2}+\left(\bar{b}_{12}-\bar{a}_{02}\right) v+\bar{b}_{03}\right)+o(r) .
\end{aligned}\right.
$$

Putting $v-1=z$ we traslate the singularity at $(r, z)=(0,0)$. The linear part of the new vector field at $(0,0)$ is

$$
\left(\left(\left(\bar{b}_{30}+\bar{b}_{21}+\bar{b}_{12}+\bar{b}_{03}\right)-\left(\bar{a}_{20}+\bar{a}_{11}+\bar{a}_{02}\right)\right) r-\left(\bar{a}_{10}-\bar{b}_{20}\right) z\right) \frac{\partial}{\partial z} .
$$

The singularity is hyperbolic for the restriction to $\{r=0\}$ and there exists a center manifold $W^{c}$ tangent to the eigenspace associated to the 0 eigenvalue. A simple calculation shows that a first order approximation of $W^{c}$ is given by

$$
\frac{\bar{a}_{20}+\bar{a}_{11}+\bar{a}_{02}-\bar{b}_{30}-\bar{b}_{21}-\bar{b}_{12}-\bar{b}_{03}}{\bar{b}_{20}-\bar{a}_{10}} r+o(r)
$$

and the corresponding reduction of the vector field to $W^{c}$ by

$$
r^{\prime}=\frac{\bar{b}_{20}\left(\bar{a}_{20}+\bar{a}_{11}+\bar{a}_{02}\right)-\bar{a}_{10}\left(\bar{b}_{30}+\bar{b}_{21}+\bar{b}_{12}+\bar{b}_{03}\right)}{\bar{b}_{20}-\bar{a}_{10}} r^{2}+o\left(r^{2}\right) .
$$

In Figure 3.7 we represent the phase-portraits for the blowing-up and the corresponding topological types for $X_{R}$ which are obtained.

Case 5: We assume that $\bar{b}_{20}>0$. Blowing-up in the $\bar{y}$-direction with $v=1$ we get

$$
\left\{\begin{array}{l}
r^{\prime}=r\left(\bar{b}_{20}+\bar{b}_{11} u\right)+o(r) \\
u^{\prime}=\left(\bar{a}_{01}-\bar{b}_{11}\right) u^{2}+u(O(r)) .
\end{array}\right.
$$

On the invariant line $\{r=0\}$ we only find a singularity at $(r, u)=(0,0)$ with linear part $\bar{b}_{20} r \frac{\partial}{\partial r}$. Therefore $\{r=0\}$ is a center manifold and the corresponding restriction is given by $\left(\bar{a}_{01}-\bar{b}_{11}\right) u^{2} \frac{\partial}{\partial u}$. We know from Remark 3.1 that $\left(\bar{a}_{01}-\bar{b}_{11}\right) \neq 0$.

Blowing-up in the $\bar{x}$-direction with $u=1$ we get

$$
\left\{\begin{aligned}
r^{\prime}= & r\left(\bar{a}_{10} v+\bar{a}_{01}\right)+o(r) \\
v^{\prime}= & -\left(\bar{a}_{01}-\bar{b}_{11}\right) v+r\left(\left(\bar{b}_{30}-\bar{a}_{20}\right) v^{3}\right. \\
& \left.+\left(\bar{b}_{21}-\bar{a}_{11}\right) v^{2}+\left(\bar{b}_{12}-\bar{a}_{02}\right) v+\bar{b}_{03}\right)+o(r) .
\end{aligned}\right.
$$


On the invariant line $\{r=0\}$ we get an extra hyperbolic singularity at $(r, v)=(0,0)$ with linear part $\bar{a}_{01} r \frac{\partial}{\partial r}+\left(\bar{b}_{03} r-\left(\bar{a}_{01}-\bar{b}_{11}\right) v\right) \frac{\partial}{\partial v}$. Glueing the different charts together we get the phase-portraits for the blowingup and for the topological types for $X_{R}$ which are depicted in Figure 3.8.
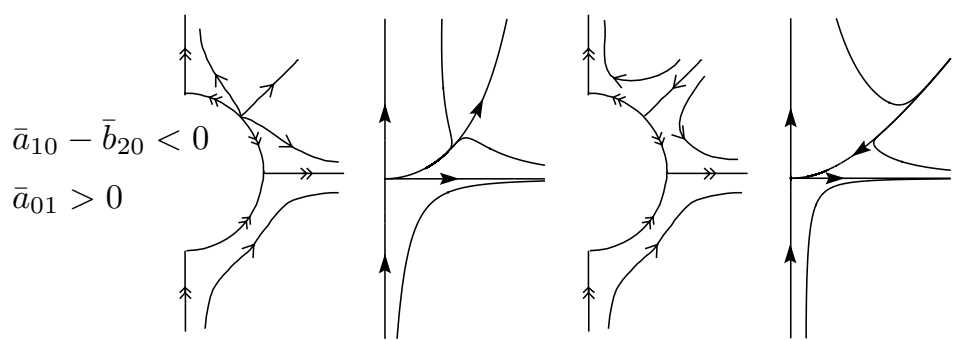

$\left(\bar{a}_{10}, \bar{a}_{20}, \bar{b}_{20}\right)=(-1,1,1) \quad\left(\bar{a}_{10}, \bar{a}_{20}, \bar{b}_{20}\right)=(-1,-1,1)$
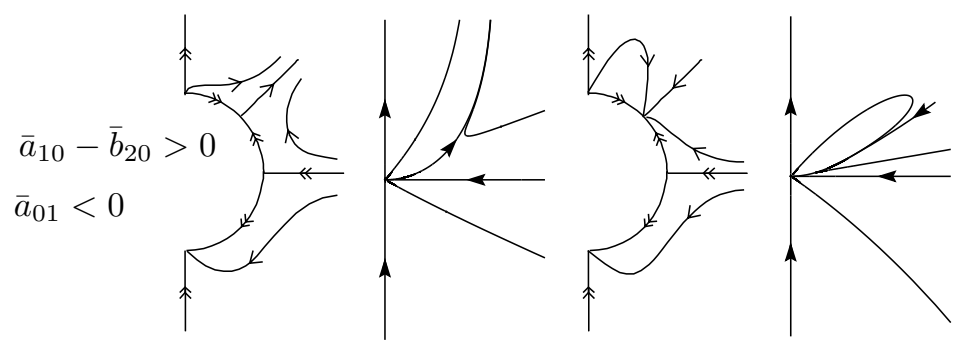

$\left(\bar{a}_{10}, \bar{a}_{20}, \bar{b}_{20}\right)=(2,-1,1) \quad\left(\bar{a}_{10}, \bar{a}_{20}, \bar{b}_{20}\right)=(2,1,1)$
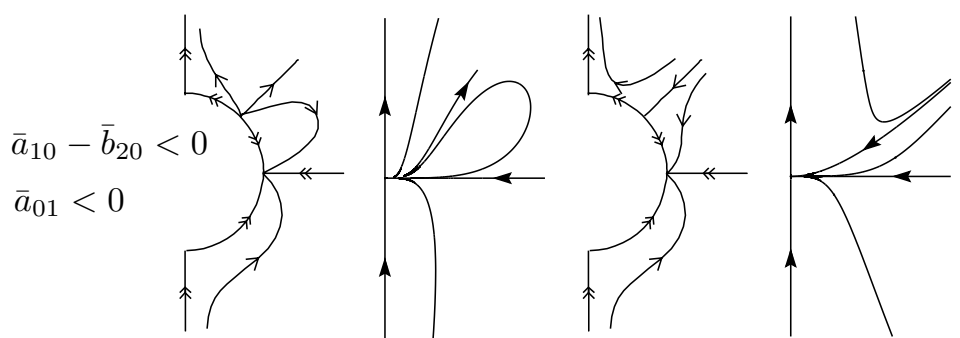

$$
\begin{array}{cc}
\left(\bar{a}_{10}, \bar{a}_{20}, \bar{b}_{20}\right)=(1 / 2,1,1) & \left(\bar{a}_{10}, \bar{a}_{20}, \bar{b}_{20}\right)=(1 / 2,-1,1) \\
\beta>0 & \beta<0
\end{array}
$$

Figure 3.7. Topological types for vector fields in $C_{4,4} \backslash C_{5,7}$. $\beta=\left(\bar{b}_{20}\left(\bar{a}_{20}+\bar{a}_{11}+\bar{a}_{02}\right)-\bar{a}_{10}\left(\bar{b}_{30}+\bar{b}_{21}+\bar{b}_{12}+\bar{b}_{03}\right)\right) /\left(\bar{b}_{20}-\bar{a}_{10}\right)$. We use the model $\frac{1}{2} x\left(\bar{a}_{10} y-\bar{a}_{10} x^{2}+\bar{a}_{20} y^{2}+\bar{a}_{11} y x^{2}+\bar{a}_{02} x^{4}\right) \frac{\partial}{\partial x}+$ $\left(\bar{b}_{20} y^{2}-\bar{b}_{20} y x^{2}+\bar{b}_{02} x^{4}+\bar{b}_{30} y^{3}+\bar{b}_{21} y^{2} x^{2}+\bar{b}_{12} y x^{4}+\bar{b}_{03} x^{6}\right) \frac{\partial}{\partial y}$. 

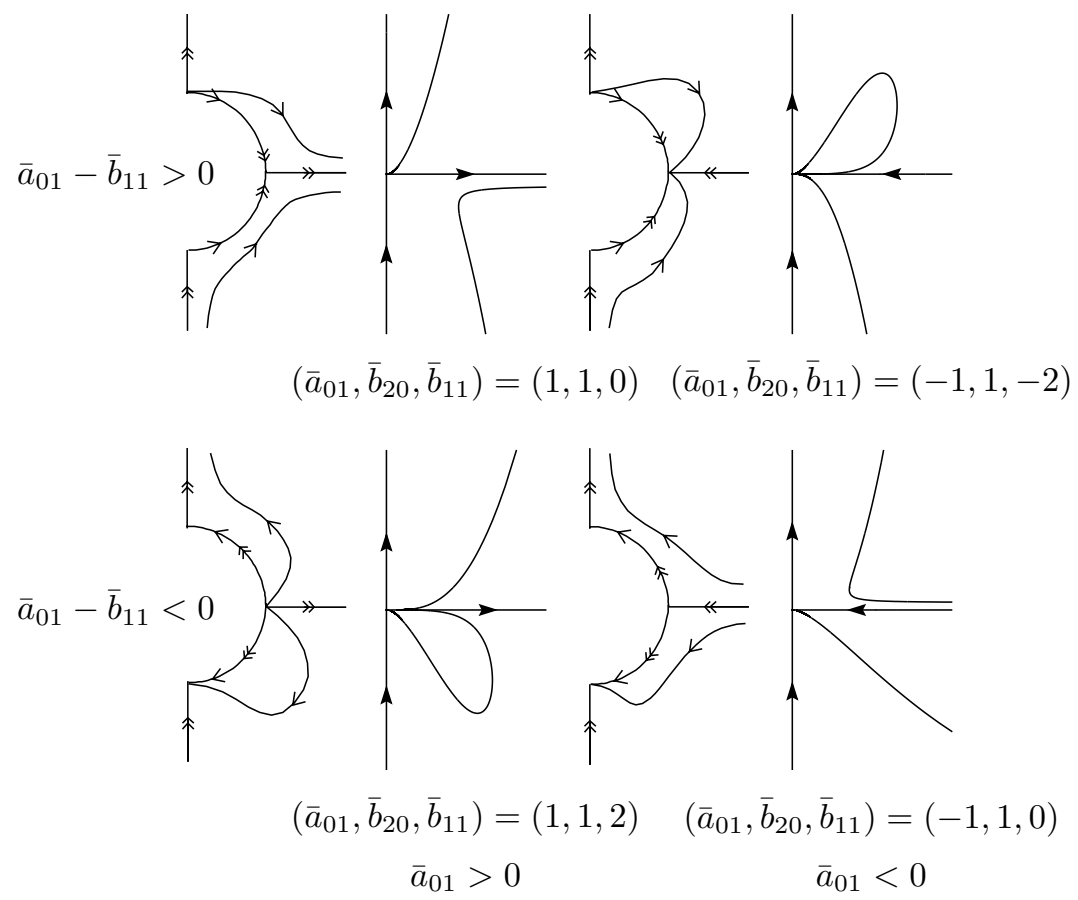

Figure 3.8. Topological types for vector fields in $C_{4,5} \backslash\left(C_{5,8} \cup C_{5,9}\right)$.

We use the model $\frac{1}{2} x\left(\bar{b}_{20} y+\bar{a}_{01} x^{2}\right) \frac{\partial}{\partial x}+\left(\bar{b}_{20} y^{2}+\bar{b}_{11} y x^{2}\right) \frac{\partial}{\partial y}$.

Case 6: Let us recall from Remark 3.1 that $\bar{a}_{01}-\bar{b}_{11}=0$. Once more again, we assume that $\bar{b}_{20}>0$. Blowing-up in the $\bar{y}$-direction with $v=1$ we get

$$
\left\{\begin{array}{l}
r^{\prime}=r\left(\bar{b}_{20}+\bar{b}_{11} u\right)+o(r) \\
u^{\prime}=u\left(\bar{a}_{10}-\bar{b}_{20}\right)+u(O(r)) .
\end{array}\right.
$$

On the invariant line $\{r=0\}$ we find exactly one hyperbolic singularity at $(r, u)=(0,0)$ with linear part $\bar{b}_{20} r \frac{\partial}{\partial r}+\left(\bar{a}_{10}-\bar{b}_{20}\right) u \frac{\partial}{\partial u}$.

Blowing-up in the $\bar{x}$-direction with $u=1$ we get

$$
\left\{\begin{aligned}
r^{\prime}= & r\left(\bar{a}_{10} v+\bar{a}_{01}\right)+o(r) \\
v^{\prime}= & \left(\bar{b}_{20}-\bar{a}_{10}\right) v^{2}+r\left(\left(\bar{b}_{30}-\bar{a}_{20}\right) v^{3}\right. \\
& \left.+\left(\bar{b}_{21}-\bar{a}_{11}\right) v^{2}+\left(\bar{b}_{12}-\bar{a}_{02}\right) v+\bar{b}_{03}\right)+o(r) .
\end{aligned}\right.
$$


Again $(r, v)=(0,0)$ is the unique singularity on $\{r=0\}$ and the linear part is given by $\bar{a}_{01} r \frac{\partial}{\partial r}+\bar{b}_{03} r \frac{\partial}{\partial v}$. Therefore $\{r=0\}$ is a center manifold and the restriction of the vector field is given by $\left(\bar{b}_{20}-\bar{a}_{10}\right) v^{2} \frac{\partial}{\partial v}$. Glueing the different charts together we get the phase-portraits depicted in Figure 3.9 where the different topological types corresponding to $X_{R}$ are also shown.
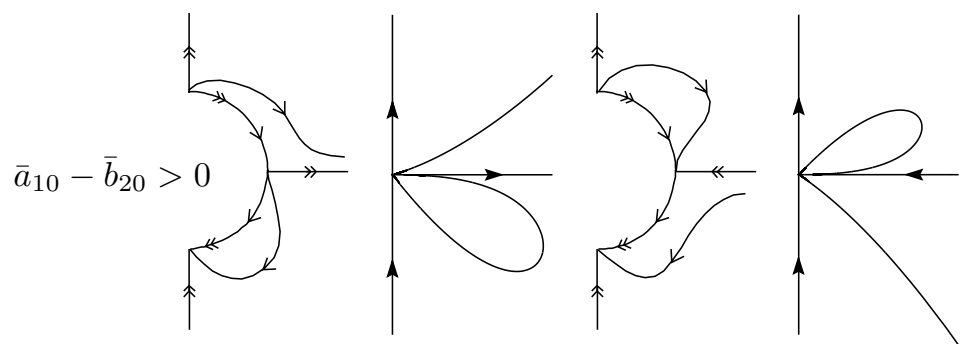

$$
\left(\bar{a}_{10}, \bar{a}_{01}, \bar{b}_{20}\right)=(2,1,1) \quad\left(\bar{a}_{10}, \bar{a}_{01}, \bar{b}_{20}\right)=(2,-1,1)
$$
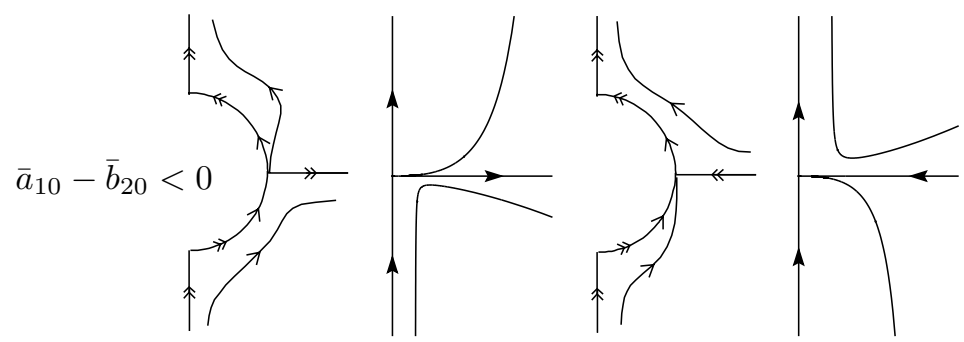

$$
\begin{array}{cc}
\left(\bar{a}_{10}, \bar{a}_{01}, \bar{b}_{20}\right)=(0,1,1) & \left(\bar{a}_{10}, \bar{a}_{01}, \bar{b}_{20}\right)=(0,-1,1) \\
\bar{a}_{01}>0 & \bar{a}_{01}<0
\end{array}
$$

Figure 3.9. Topological types for vector fields in $C_{4,6} \backslash\left(C_{5,10} \cup C_{5,11}\right)$. We use the model $\frac{1}{2} x\left(\bar{a}_{10} y+\bar{a}_{01} x^{2}\right) \frac{\partial}{\partial x}+\left(\bar{b}_{20} y^{2}+\bar{a}_{01} y x^{2}\right) \frac{\partial}{\partial y}$.

\section{References}

[CH] S. -N. ChOW AND J. HALE, "Methods of bifurcation theory," Springer-Verlag, New York, Berlin, Heidelberg, 1982.

[D] F. Dumortier, Singularities of vector fields on the plane, J. Differential Equations 23 (1977), 53-106.

[DI] F. DUmortier AND S. IBÁÑEz, Singularities of vector fields on $\mathbb{R}^{3}$, Nonlinearity 11 (1998), 1037-1047. 
[DH] F. Dumortier And C. Herssens, Tracing phase portraits of planar polynomial vector fields with detailed analysis of the singularities, Preprint (1998).

[H] C. Herssens, Drawing and classifying portraits of planar polynomial vector fields, Thesis, Limburgs Universitair Centrum (1998).

[KR] B. Krauskopf and C. Rousseau, Codimension-three unfoldings of reflectionally symmetric planar vector fields, Nonlinearity $\mathbf{1 0}$ (1997), 1115-1150.

[T] F. TAKEns, Singularities of vector fields, Inst. Hautes Études Sci. Publ. Math. 43 (1974), 47-100.

\author{
F. Dumortier: \\ Limburgs Universitair Centrum \\ Universitaire Campus \\ B-3590 Diepenbeek \\ BELGIUM
}

e-mail: fdumorti@luc.ac.be

\author{
S. Ibáñez: \\ Departamento de Matemáticas \\ Universidad de Oviedo \\ 33007 Oviedo \\ SPAIN
}

e-mail: mesa@pinon.ccu.uniovi.es

Primera versió rebuda el 25 de juny de 1998 , darrera versió rebuda el 27 de gener de 1999 